\title{
Can weakly nonlinear theory explain Faraday wave patterns near onset?
}

\author{
A. C. SKELD O N $\mathrm{N}^{1}$, and A. M. RUCKLID G E $\mathbf{E}^{2}$ \\ ${ }^{1}$ Department of Mathematics, University of Surrey, Guildford, Surrey, GU2 7XH, UK \\ ${ }^{2}$ Department of Mathematics, University of Leeds, Leeds, LS2 9JT, UK \\ (Received ?; revised ?; accepted ?. - To be entered by editorial office)
}

The Faraday problem is an important pattern-forming system that provides some middle ground between systems where the initial instability involves just a single mode and in which complexity then results from mode interactions or secondary bifurcations, and cases where a system is highly turbulent and many spatial and temporal modes are excited. It has been a rich source of novel patterns and of theoretical work aimed at understanding how and why such patterns occur. Yet it is particularly challenging to tie theory to experiment: the experiments are difficult to perform; the parameter regime of interest (large box, moderate viscosity) along with the technical difficulties of solving the free boundary Navier-Stokes equations make numerical solution of the problem hard; and the fact that the instabilities result in an entire circle of unstable wavevectors presents considerable theoretical difficulties.

In principle, weakly nonlinear theory should be able to predict which patterns are stable near pattern onset. In this paper we present results from a quantitative comparison between weakly nonlinear theory of the Navier-Stokes equations and previously published experimental results for the Faraday problem. The extent to which proposed theoretical mechanisms that show how three-wave interactions can stabilise complex patterns, including quasipatterns, are supported by the comparison is discussed and discrepancies are highlighted.

Key words: Faraday waves, superlattice patterns, quasipatterns

\section{Introduction}

Since Faraday (1831) identified that regular patterns can appear on the surface of a shaken container of fluid, the Faraday experiment has been an important system for investigating pattern formation. Experiments in the 1980's such as those conducted by Simonelli \& Gollub (1989) tended to focus on the dynamics of the interaction of patterns in small containers excited by a sinusoidal forcing with a single frequency component. More recently the focus has switched to larger containers with multiple frequency components: as pointed out in Arbell \& Fineberg (1998), one special feature of the Faraday experiment is that by using multiple frequency forcing one can investigate the interaction of a small number of controllable modes with different characteristic length scales. This provides some middle ground between pattern-forming systems where the initial instability involves just a single mode and in which complexity then results from secondary bifurcations, such as in the Bénard-Marangoni experiment or the Taylor-Couette

$\dagger$ Email address for correspondence: a.skeldon@surrey.ac.uk 
experiment, and cases where a system is highly turbulent and many spatial and temporal modes are excited.

Within the Faraday experiment, a rich variety of complex patterns are seen, some of which have a complicated spatial structure but are time-periodic with the periodicity of the drive and some with both a complicated spatial and temporal structure. Aside from the ubiquitous stripes, squares and hexagons, observed patterns include: quasipatterns (Christiansen et al. (1992), Edwards \& Fauve (1994)); superlattice patterns (SLI) (Kudrolli et al. (1998); Epstein \& Fineberg (2006)); spatially subharmonic superlattice states, modulated hexagonal disorder, two mode superlattices and unlocked states (Arbell \& Fineberg (1998)); oscillons (Arbell \& Fineberg (2000)), and double hexagon states Arbell \& Fineberg (1998). An excellent summary of many of the experimental results is given in Arbell \& Fineberg (2002).

In parallel with the experiments, theoretical advances have resulted in an effective numerical method for performing the linear stability analysis of the Navier-Stokes equation that marks the transition from an unpatterned to patterned state (Kumar \& Tuckerman (1994)). The challenge here is that many of the experiments are carried out at moderate viscosity whereas early theoretical results of Benjamin \& Ursell (1954), which help explain the underlying instability mechanism, are for an inviscid fluid. There have also been theoretical explanations for why, unlike the Bénard-Marangoni system, the observed patterned states include superlattice patterns (Silber \& Skeldon (1999); Silber et al. (2000); Silber \& Proctor (1998); Rucklidge \& Silber (2009)).

These theoretical mechanism rely on three-wave resonance between critical modes and modes close to critical and have successfully explained why particular superlattice patterns are observed. The suggested theoretical mechanism for the appearance of superlattice patterns is compelling, and the link between which modes are excited and the patterns that are observed has been explored in some detail experimentally, for example see Arbell \& Fineberg (2002); Epstein \& Fineberg (2006). However, without carrying out a careful quantitative comparison between experiment and theory, it is hard to know the extent to which the theoretical ideas really do explain the experimental results $(\overline{\mathrm{Bo}-}$ denschatz et al. (2000)). This is particularly true for the Faraday experiment where, as we will explain in greater detail below, the very region for which the theory predicts superlattice patterns is the region for which some of the underlying assumptions of the theory break down.

While it is not possible to write down closed-form solutions of the full Navier-Stokes equations for the Faraday problem, quantitative predictions of the patterns expected near the transition from non-patterned to pattern states can be made using weakly nonlinear analysis (Skeldon \& Guidoboni (2007)). Weakly nonlinear analysis centres on using an asymptotic expansion in terms of the slowly varying amplitudes of the critical modes at onset, using ideas first developed in Malkus \& Veronis (1958) and Segel \& Stuart (1962) in the context of convection experiments. Analysis of the resulting amplitude equations leads to predictions on the relative stability of different patterned states.

Our aim in this paper is to do a quantitative comparison between previously published experimental results and a weakly nonlinear analysis of the Navier-Stokes equations for the Faraday problem. We will discuss to what extent the existing weakly nonlinear theory can explain observed patterns and provide some new explanations in some cases. While agreement is very good in many cases, we note that quantitatively linking theory with experiment is particularly challenging after-the-fact as the results are sensitive to precise values of viscosity and surface tension and even the sign of the drive term, something that is not normally recorded. There are also places where the analysis strongly suggest that patterns should have a subharmonic component, when no subharmonic component 
has been observed. In particular, with regard to the superlattice patterns, we discuss two methods that have been used to promote the stability of superlattice patterns: firstly by approaching the so-called bicritical point in two-frequency forced experiments; and, secondly, by adding a third frequency to the drive. We highlight the differences between these two mechanisms.

\section{Equations}

Using a variety of container shapes, Edwards \& Fauve (1994) elegantly demonstrated that many of the patterns that occur with moderate viscosity fluids in large containers are not strongly dependent on the lateral boundaries of the container. Consequently it is a reasonable modelling assumption to consider an infinite horizontal layer of viscous incompressible fluid of finite depth that is subjected to gravity $g$ and to a vertical periodic excitation with frequency components $j \tilde{\omega}$. At the lower boundary the fluid is in contact with a rigid plane while at the upper boundary the surface is open to the atmosphere. This means that the upper surface is a free boundary whose shape and evolution is an unknown of the problem.

The motion of the fluid can be described by the Navier-Stokes equations, where, to take account of the parametric excitation, a frame of reference which is moving with the periodic excitation is considered. The $z$-axis is chosen perpendicular to the rigid plane at the bottom, which lies at $z=-h / l$, where $h / l$ is the non-dimensional depth of the layer when the fluid is at rest. A sketch of the geometry is shown in figure 1 . Assuming the free surface may be written as $z=\zeta(x, y ; t)$, which excludes the formation of droplets or breaking waves, then the fluid motion in the bulk is described by the dimensionless incompressible Navier-Stokes equations

$$
\begin{aligned}
\nabla \cdot \mathbf{u} & =0 \\
\partial_{t} \mathbf{u}+\mathbf{u} \cdot \nabla \mathbf{u} & =-\nabla \mathcal{P}+C \Delta \mathbf{u}-(1+f(t)) \mathbf{e}_{3},
\end{aligned}
$$

where $\mathbf{u}=(u, v, w)$ is the velocity field, $\mathcal{P}$ the pressure and for multiple frequency excitation,

$$
f(t)=\sum_{j} \tilde{a}_{j} \cos \left(j \tilde{\omega} t+\phi_{j}\right)
$$

where $j$ are integers and $\tilde{a}_{j}$ and $\phi_{j}$ are real.

It is assumed that the bottom of the container is rigid so that at $z=-h / l$ the fluid satisfies the no-slip boundary conditions

$$
u=v=w=0 .
$$

At the free surface $z=\zeta(x, y ; t)$ we have the kinematic condition, which says that the surface is advected by the fluid, and two further conditions, one for the balance of the tangential stresses and one for the balance of normal stresses. This leads to three conditions at $z=\zeta(x, y ; t)$ namely

$$
\begin{aligned}
\partial_{t} \zeta+u \partial_{x} \zeta+v \partial_{y} \zeta & =w \\
\mathbf{t}_{1} \cdot \mathbf{T n}=\mathbf{t}_{2} \cdot \mathbf{T n} & =0 \\
-\mathcal{P}+2 C \mathbf{n D}(\mathbf{u}) \mathbf{n} & =B \mathcal{H}-p_{e},
\end{aligned}
$$

where $\mathbf{T}=-\mathcal{P} \mathbf{I}+2 C \mathbf{D}(\mathbf{u})$ is the stress tensor, $\mathbf{D}(\mathbf{u})=\left(\nabla \mathbf{u}+\nabla^{T} \mathbf{u}\right) / 2$ is the rate-ofstrain tensor, and $\mathcal{H}=\nabla_{H} \cdot\left(\nabla_{H} \zeta / \sqrt{1+\left|\nabla_{H} \zeta\right|^{2}}\right)$ is the double mean curvature. Note that $\nabla=\left(\nabla_{H}, \partial_{z}\right)$ and $\nabla_{H}=\left(\partial_{x}, \partial_{y}\right)$. The unit normal and tangent vectors are defined 


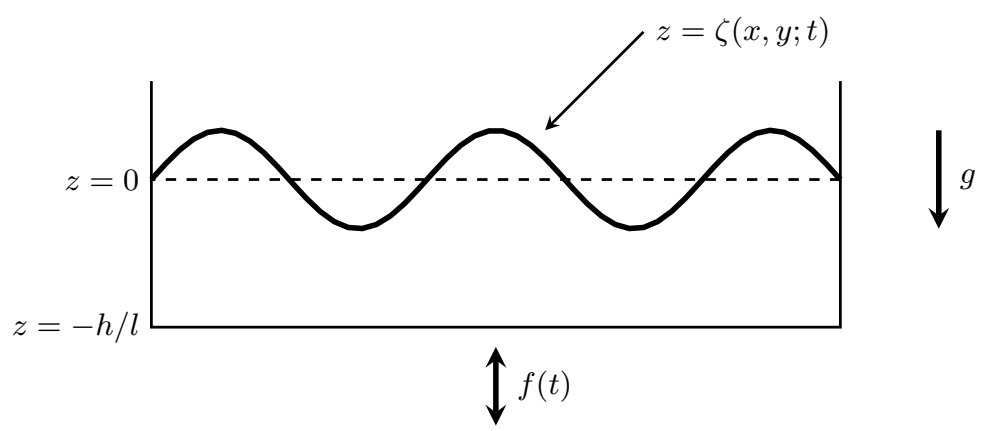

FIGURE 1. Sketch of a cross-section through the layer of fluid.

as

$$
\begin{aligned}
& \mathbf{n}(x, y ; t)=\left(-\frac{\partial_{x} \zeta}{\sqrt{1+\left|\nabla_{H} \zeta\right|^{2}}},-\frac{\partial_{y} \zeta}{\sqrt{1+\left|\nabla_{H} \zeta\right|^{2}}}, \frac{1}{\sqrt{1+\left|\nabla_{H} \zeta\right|^{2}}}\right), \\
& \mathbf{t}_{1}(x, y ; t)=\left(\frac{1}{\sqrt{1+\left|\partial_{x} \zeta\right|^{2}}}, 0, \frac{\partial_{x} \zeta}{\sqrt{1+\left|\partial_{x} \zeta\right|^{2}}}\right), \\
& \mathbf{t}_{2}(x, y ; t)=\left(0, \frac{1}{\sqrt{1+\left|\partial_{y} \zeta\right|^{2}}}, \frac{\partial_{y} \zeta}{\sqrt{1+\left|\partial_{y} \zeta\right|^{2}}}\right),
\end{aligned}
$$

The units of length, time, velocity and pressure have been taken as $l, \sqrt{l / g}, \sqrt{g l}$ and $\varrho g l$ respectively. The length scale $l$ is taken to be a length scale that is typical for the problem, such as the primary wavelength of the observed patterns. Here, $p_{e}$ is the dimensionless pressure of the external ambient fluid and is assumed known. There are two non-dimensional parameters associated with the fluid, namely: $C=\nu /\left(g l^{3}\right)^{1 / 2}$, the square of the inverse of the Galileo number, and $B=\sigma / \varrho g l^{2}$, the inverse Bond number, where $\nu$ is the kinematic viscosity, $\sigma$ is the surface tension and $\varrho$ is the density of the fluid. These two non-dimensional parameters measure the relative importance of viscous and surface tension forces compared to gravity respectively. There are three other sets of nondimensional parameters of importance, all associated with the excitation. These are: the non-dimensional amplitudes $\tilde{a}_{j}$, frequencies $j \tilde{\omega}$ and phases $\phi_{j}$ of the components of the excitation.

As in Kumar \& Tuckerman (1994), it is convenient to define a new pressure,

$$
p=\mathcal{P}+(1+f(t)) z,
$$

which has the effect of shifting the acceleration term from the momentum equation to the normal stress condition. In addition, we eliminate the pressure from the momentum equation by taking $-(\nabla \times \nabla \times)$. Using the relation $\nabla \times \nabla \times \mathbf{u}=\nabla(\nabla \cdot \mathbf{u})-\Delta \mathbf{u}$ and the fact that $\nabla \cdot \mathbf{u}=0$, the problem then becomes

$$
\begin{aligned}
\nabla \cdot \mathbf{u} & =0 \\
\partial_{t} \Delta \mathbf{u}-C \Delta \Delta \mathbf{u} & =\nabla \times \nabla \times(\mathbf{u} \cdot \nabla \mathbf{u}),
\end{aligned}
$$


with boundary conditions on $z=-h / l$,

$$
u=v=w=0
$$

and on $z=\zeta$,

$$
\begin{aligned}
\partial_{t} \zeta+u \partial_{x} \zeta+v \partial_{y} \zeta & =w, \\
\mathbf{t}_{1} \cdot \mathbf{T n}=\mathbf{t}_{2} \cdot \mathbf{T n} & =0, \\
2 C \mathbf{n D}(\mathbf{u}) \mathbf{n} & =B \mathcal{H}+p-p_{e}-(1+f(t)) \zeta .
\end{aligned}
$$

Equations 2.6 with boundary conditions 2.7$)$ and $(2.8)$ have a trivial solution,

$$
\mathbf{u}=\mathbf{0}, \quad p=p_{e}, \quad \zeta=0 .
$$

This solution corresponds to an unpatterned state where there is no relative motion of the fluid with respect to the moving frame so the surface of the fluid is flat.

\section{Does linear theory agree with experiments?}

Understanding the linear stability of the unpatterned state is at the heart of understanding many of the nonlinear patterns that are formed close to onset. This is because when the unpatterned state becomes unstable to one critical mode with a given critical wavenumber, there are often several other modes with different wavenumbers that are themselves only weakly damped. This is particularly true with multi-frequency forcing. Resonant interaction of instabilities from different critical/close to critical modes drive the selection mechanisms for the occurrence of particular patterns (Silber et al. (2000)).

Benjamin \& Ursell (1954) recognised that the linear stability of the flat-surface solution for an inviscid, infinite depth fluid driven by a single frequency reduces to a Mathieu equation. The Mathieu equation contains two parameters, related to the frequency $\tilde{\omega}$ and amplitude $\tilde{a}$ of the excitation respectively. Solutions to the Mathieu equation divide the parameter plane into regions of bounded and regions of unbounded solutions where the regions of unbounded solutions form tongues that touch the frequency axis at frequencies $m \tilde{\omega} / 2, m=1,2 \ldots$, the largest tongue occuring for $m=1$. The tongues are typically classified as either harmonic or subharmonic, depending on whether or not they are an integer multiple of the frequency $\tilde{\omega}$. This picture is modified with the addition of damping: the boundaries of the regions are perturbed and no longer touch the frequency axis, consequently a finite amplitude of excitation is required to excite waves; the regions of bounded solutions become regions where the unpatterned state is locally stable; the unbounded regions become regions where the unpatterned state is locally unstable.

Kumar \& Tuckerman (1994) identified a numerical method to find the instability tongues that can be used for all fluid viscosities and all depths and applied it to the case of single frequency excitation. This was extended to multiple frequency excitation by Besson et al. (1996). A typical example of tongues computed using Besson et al. (1996)'s method and the corresponding bifurcation set for the primary stability boundary is shown in figure 2 .

The linear stability problem appears to be solved: the numerical method works well and in Besson et al. (1996) the authors show that there is excellent agreement between their linear stability calculations and experiments. However, unless experiments and theory are carried out hand-in-hand, in practice there remain some difficulties in obtaining really good agreement between theory and experiment even for this first transition from patterned to unpatterned state. This is illustrated in figure 3 where numerical linear stability results are superimposed on the experimental bifurcation sets published in: 
(a)

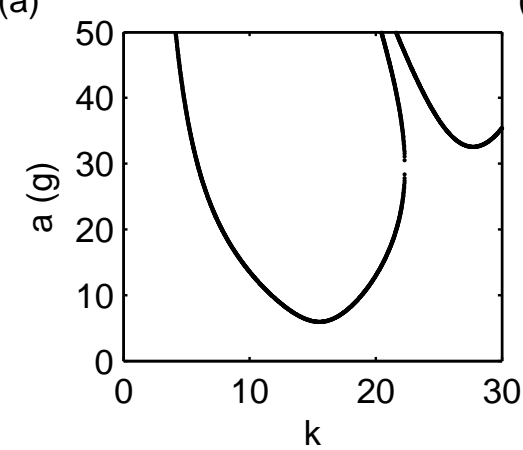

(c)

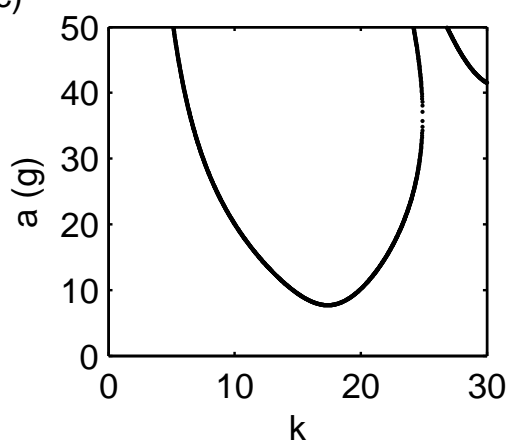

(b)
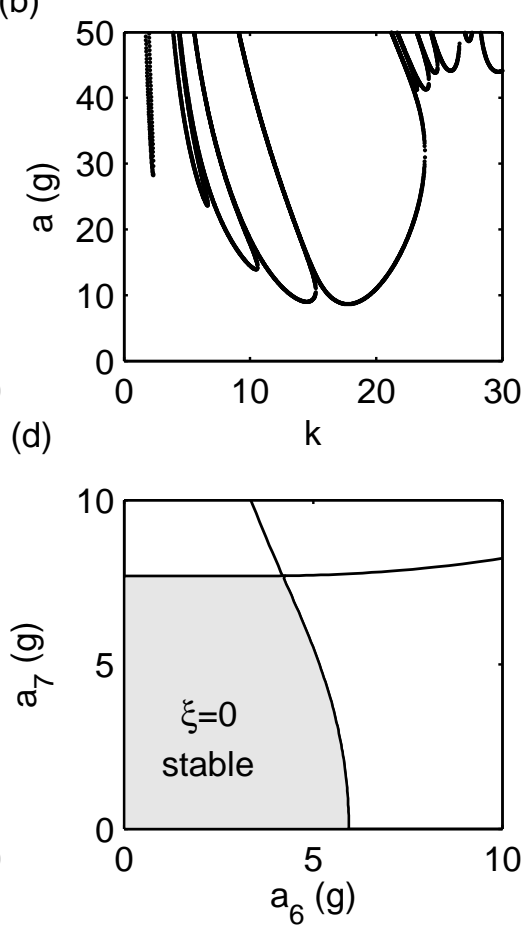

FiguRE 2. Typical tongues and linear stability region for the unpatterned state computed using the method of Besson et al. (1996). Parameters as for Kudrolli et al. (1998): the (dimensional) excitation is $f_{d}(t)=a_{6} \cos 6 \omega t+a_{7} \cos \left(7 \omega t+\phi_{7}\right)$, where $a_{6}=a \cos \chi, a_{7}=a \sin \chi, \phi_{7}=20^{\circ}$, $\omega / 2 \pi=16.44 \mathrm{~Hz}, \sigma=20.6 \mathrm{dyn} \mathrm{cm}^{-1}, \nu=0.20 \mathrm{~cm}^{2} \mathrm{~s}^{-1}, \rho=0.95 \mathrm{~g} \mathrm{~cm}^{-3}, h=0.3 \mathrm{~cm}$. Note that the structure inherited from the Mathieu equation is seen by the fact that the minimum of each tongue occurs at half integer multiples of the drive frequency. In (a) $\chi=0^{\circ}$, the forcing reduces to $f_{d}(t)=a_{6} \cos 6 \omega t$, with a forcing frequency of $6 \omega$. The largest tongue has a minimum for wavenumber of approx $15 \mathrm{~cm}^{-1}$ for a forcing amplitude $a$ of approx $7.5 \mathrm{~g}$ and corresponds to a mode of frequency $\frac{6 \omega}{2}$. Whereas in (c), $\chi=90^{\circ}$ the forcing reduces to $f_{d}(t)=a_{7} \cos 7 \omega t$ with a forcing frequency of $7 \omega$ leading to a primary instability mode with frequency $7 \omega / 2$. In the case of (b), $\chi=63^{\circ}$, the presence of both $6 \omega$ and $7 \omega$ components in the forcing frequency mean that the drive has periodicity $2 \pi / \omega$ and is therefore of frequency $\omega$. This leads to tongues at $m \omega / 2 \ldots, m=1,2, \ldots$. The first five tongues that are visible from left to right correspond to: $\omega / 2,4 \omega / 2,5 \omega / 2,6 \omega / 2,7 \omega / 2$. The largest tongues correspond to the $6 \omega / 2$ and the $7 \omega / 2$ modes and are driven by the two main frequency components of the drive. There are tongues corresponding to modes with frequency $2 \omega / 2$ and $3 \omega / 2$ but these occur off the top of the region shown. This particular value of $\chi$ is close to the 'bi-critical' point where both harmonic modes with frequency $6 \omega / 2$ and sub-harmonic modes with frequency $7 \omega / 2$ onset simultaneously. It is close to this bi-critical point that many of the exotic patterns are observed. (d) Bifurcation set showing the position of the tongue minimum that marks the instability of the unpatterned state, as a function of $a_{6}$ and $a_{7}$.

Edwards \& Fauve (1994) (panels (a) and (b)); Kudrolli et al. (1998) (panel (c)); Epstein \& Fineberg (2006) (panel (d)), and Ding \& Umbanhowar (2006) (panels (e) and (f)).

The central issue here is that the position of the curves is sensitive to the values of the surface tension, density and the viscosity and yet the values quoted in papers are often taken from the manufacturers' specifications for the fluids used. In order to illustrate the issue, in each case we have plotted the linear stability curves for the quoted viscosity and the quoted viscosity plus or minus $5 \%$, a typical quoted tolerance for the 
viscosity value. From figure 3 we see that: for the experiments in Edwards \& Fauve (1994) and Ding \& Umbanhowar (2006), the upper extreme for the viscosity fits the data best (panels (a),(b),(e) and (f)); for those in Kudrolli et al. (1998), the lower extreme fits the subharmonic boundary best, but not the harmonic boundary (panel (c)); for the experiments in Epstein \& Fineberg (2006) the linear stability analysis suggests that the actual viscosity of the fluid was higher than the upper value (panel (d)). We have focussed here on viscosity because for the different fluids used in the results presented here, errors in the viscosity have the biggest effect on the linear stability boundary. A error in the viscosity of $5 \%$ can result in an error in the linear stability boundary of $5 \%$, whereas a $5 \%$ error in either the surface tension or the density leading to a $5 \%$ error in the ratio $\sigma / \rho$ leads to an error in the linear stability boundary of only around $1 \%$.

Our aim in this paper is to compare weakly nonlinear theory with experiments. Since the transitions from one preferred patterns to another turn out to be quite delicate, clearly it is only realistic to hope for agreement for nonlinear pattern selection if there is first excellent agreement with linear theory. In making our nonlinear comparisons below, we have therefore used values of the viscosity found by fitting the linear theory to the published data.

\section{Predicting patterns close to onset}

\subsection{Theoretical ideas}

Once a mode with a given wave number has become unstable, the fact that there is no preferred horizontal direction means that standing waves of any orientation can occur. In the Bénard-Marangoni experiment, which has a similar orientational invariance, this means that patterns consisting of nonlinear superpositions of modes with wave vectors of the same wave number but different orientations often lead to the observation of patterns with, for example, hexagonal symmetry. In principle, superlattice patterns could also occur in convection experiments (Skeldon \& Silber (1998)), but in practice they have not been seen without the addition of a vertical oscillation, as in Rogers et al. (2000).

The ubiquitous occurrence of hexagons is a consequence of the importance of threewave interactions in determining which patterned states occur: three-wave interactions give rise to the lowest order nonlinear (quadratic) terms in the amplitude equations that describe behaviour close to onset. For Bénard-Marangoni convection, where instability to a single dominant wavenumber occurs, the three-wave interaction of importance occurs between three wave vectors with the same critical wavenumber, as shown in figure 4(a). A distinct feature of the Faraday problem is that the Mathieu tongue-like structure to the linear stability problem means that although typically there is a single mode that becomes unstable first, there are nearby modes with different wavenumbers that are only weakly damped. This can give rise to other three-wave interactions of relevance, such as those shown in figure 4 (b), (c) and (d).

These three-wave interactions involve waves with wavevectors $\mathbf{k}_{\mathbf{1}}, \mathbf{k}_{\mathbf{2}}$ and $\mathbf{k}_{\mathbf{3}}$ and respective frequencies $n \omega / 2, p \omega / 2$ and $q \omega$. For the three waves to interact they must satisfy a spatial resonance condition,

$$
\pm \mathbf{k}_{\mathbf{1}} \pm \mathbf{k}_{\mathbf{2}}=\mathbf{k}_{\mathbf{3}}
$$

and a temporal resonance condition. This temporal resonance condition depends on both the frequencies of the waves and the various frequencies contained within the forcing term $f(t)$ and requires

$$
\pm \frac{n}{2} \pm \frac{p}{2} \pm \sum_{j} \pm j=q
$$


(a)

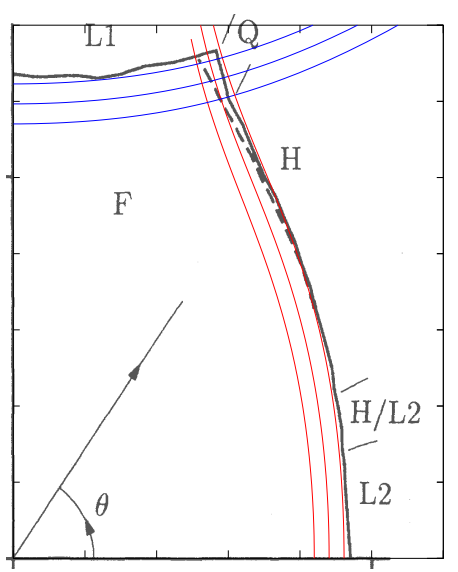

(c)

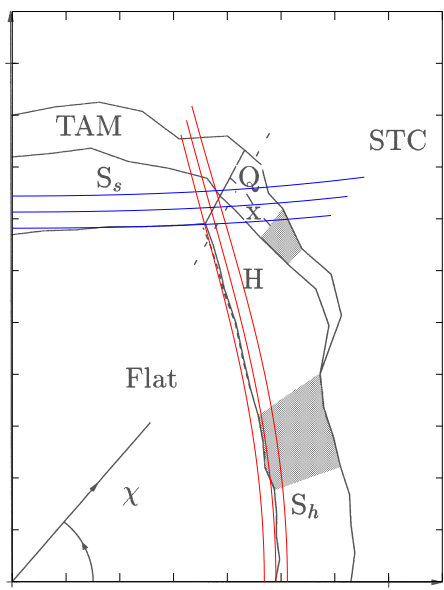

(e)

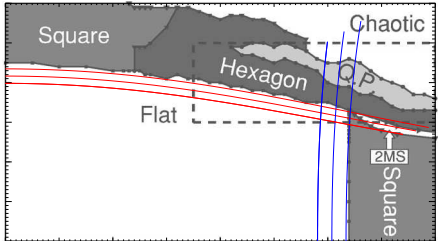

(b)

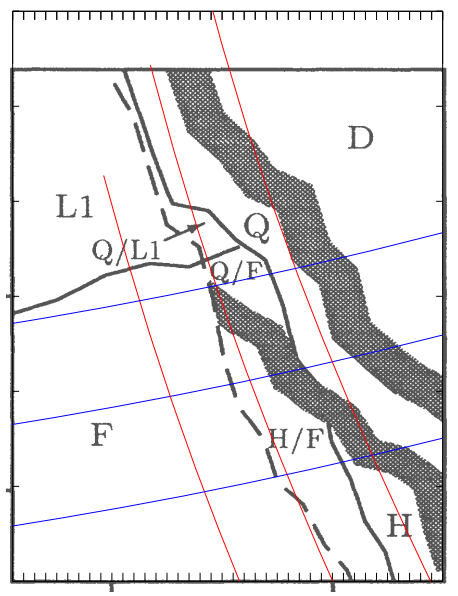

(d)

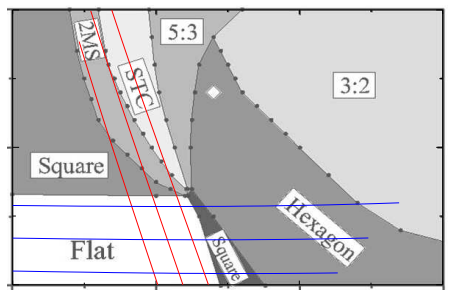

(f)

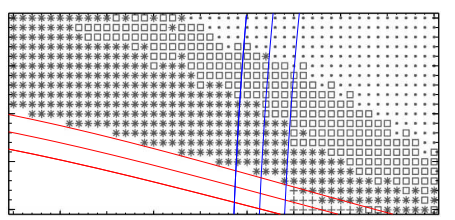

FiguRE 3. Comparison of the experimentally measured transition from unpatterned to patterned state with numerically calculated linear stability curves using the method of Besson et al. (1996). For each of the numerical calculations, three different values for the viscosity are used. (a) and (b) Numerical curves overlaid on figure 12 of Edwards \& Fauve (1994) with permission $\ldots: \sigma=65 \mathrm{dyn} \mathrm{cm}{ }^{-1} ; \nu=1.00 \pm 0.05 \mathrm{~cm}^{2} \mathrm{~s}^{-1} ; \rho=1.22 \mathrm{~g} \mathrm{~m}^{-3} ; h=0.29 \mathrm{~cm} ; a_{j}, j=\{4,5\}$; $\phi_{4}=0 ; \phi_{5}=75^{\circ} ; \omega / 2 \pi=14.6 \mathrm{~Hz}$. (c) Numerical curves overlaid on figure 6 (a) of Kudrolli et al. (1998) reprinted from Physica D, Vol 123, A. Kudrolli, B. Pier and J.P. Gollub, 'Superlattice patterns in surface waves', 99-111, Copyright (1998), with permission from Elsevier. $\sigma=20.6$ dyn $\mathrm{cm}^{-1} ; \nu=0.20 \pm 0.01 \mathrm{~cm}^{2} \mathrm{~s}^{-1} ; \rho=0.95 \mathrm{~g} \mathrm{~m}^{-3} ; h=0.3 \mathrm{~cm} ; a_{j}, j=\{4,5\} ; \phi_{4}=0 ; \phi_{5}=16^{\circ}$; $\omega / 2 \pi=22 \mathrm{~Hz}$. (d) Numerical curves overlaid on figure 2 of Epstein \& Fineberg (2006). Adapted with permission from Epstein \& Fineberg (2006). Copyrighted by the American Physical Society. $\sigma=20.6$ dyn $\mathrm{cm}^{-1} ; \nu=0.180 \pm 0.009 \mathrm{~cm}^{2} \mathrm{~s}^{-1} ; \rho=0.949 \mathrm{~g} \mathrm{~m}^{-3} ; h=0.3 \mathrm{~cm} ; a_{j}, j=\{6,7\}$; $\phi_{j}=0^{\circ} ; \omega / 2 \pi=14 \mathrm{~Hz}$. (e) and (f) Numerical curves overlaid on figures 1 and 3(a) of Ding \& Umbanhowar (2006). Adapted with permission from Ding \& Umbanhowar (2006). Copyrighted by the American Physical Society. $\sigma=20.6 \mathrm{dyn} \mathrm{cm}^{-1} ; \nu=0.20 \pm 0.01 \mathrm{~cm}^{2} \mathrm{~s}^{-1} ; \rho=0.95 \mathrm{~g}$ $\mathrm{m}^{-3} ; h=0.65 \mathrm{~cm} ; a_{j}, j=\{4,5\} ; \phi_{4}=0 ; \phi_{5}=16^{\circ} ; \omega / 2 \pi=20 \mathrm{~Hz}$. 

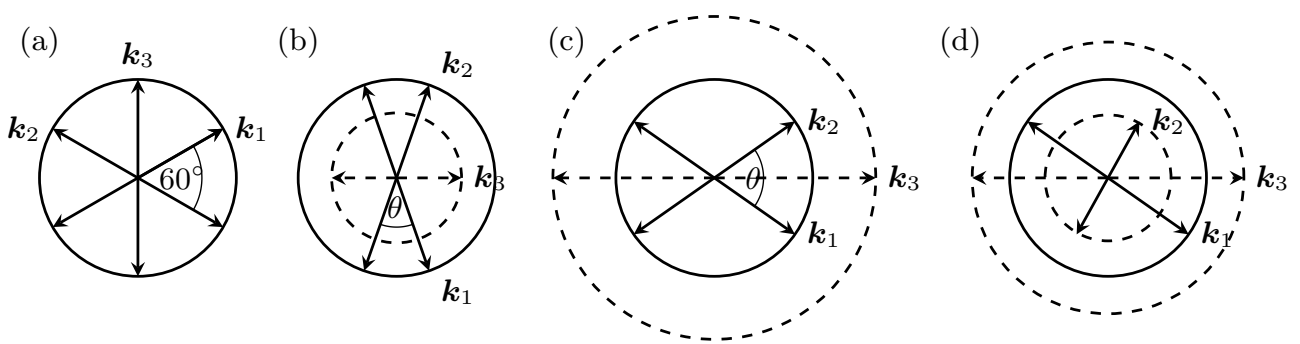

Figure 4. Possible spatial three-wave resonant interactions defined such that $k_{1}+k_{2}=k_{3}$. (a) $\left|k_{1}\right|=\left|k_{2}\right|=\left|k_{3}\right|$; (b) $\left|k_{1}\right|=\left|k_{2}\right|>\left|k_{3}\right|$; (c) $\left|k_{1}\right|=\left|k_{2}\right|<\left|k_{3}\right|$; (d) $\left|k_{1}\right| \neq\left|k_{2}\right| \neq\left|k_{3}\right|$.

where $j$ come from the forcing component of the drive, see equation 2.2 , and where all possible sign combinations of the different terms on the left-hand side need to be considered.

The fact that a temporal resonance condition needs to be satisfied is one feature that distinguishes the Faraday problem from Swift-Hohenberg multiple resonance problems such as those studied in Müller (1994), Lifshitz \& Petrich (1997) and others. This means that results from Swift-Hohenberg like equations need to be interpreted with care when applied to the Faraday problem, a point that we will return to in the discussion.

For the appearance of superlattice patterns the argument goes that, given three critical modes $\mathbf{k}_{i}$ as shown in figure 4(b) or (c) with amplitudes $A_{i}$, then using a multiple timescale expansion near onset would lead to equations for the evolution of the amplitudes on a slow timescale of the form

$$
\begin{aligned}
& \dot{A}_{1}=\lambda_{1} A_{1}+\alpha_{1} \overline{A_{2}} A_{3}+A_{1}\left(a\left|A_{1}\right|^{2}+b_{0}\left|A_{2}\right|^{2}+c\left|A_{3}\right|^{2}\right)+\ldots \\
& \dot{A}_{2}=\lambda_{1} A_{2}+\alpha_{1} \overline{A_{1}} A_{3}+A_{2}\left(b_{0}\left|A_{1}\right|^{2}+a\left|A_{2}\right|^{2}+c\left|A_{3}\right|^{2}\right)+\ldots \\
& \dot{A}_{3}=\lambda_{2} A_{3}+\alpha_{2} A_{1} A_{2}+A_{3}\left(d\left|A_{1}\right|^{2}+d\left|A_{2}\right|^{2}+e\left|A_{3}\right|^{2}\right)+\ldots,
\end{aligned}
$$

where $\lambda_{1}$ and $\lambda_{2}$ are the linear growth rates of the respective modes, and $\alpha_{1}, \alpha_{2}, a, b_{0}, c, d$ and $e$ are all real-valued constants. The quadratic coefficients, $\alpha_{1}$ and $\alpha_{2}$ are non-zero only if the temporal resonance condition is met. Note that there is no assumption of weak forcing/damping and so terms such as $\overline{A_{1}}$ do not appear Alnahdi et al. (2014).

Now, the mode with wavevector $\mathbf{k}_{\mathbf{3}}$ is not at its critical point but is weakly damped, so $\lambda_{2}<0$ and, close to onset (i.e. $\left|\lambda_{1}\right|$ small enough) it is therefore slaved by the critical modes. Consequently, one can perform a centre manifold reduction on equations 4.2, resulting in

$$
A_{3}=-\frac{\alpha_{2}}{\lambda_{2}} A_{1} A_{2}+\ldots
$$

and

$$
\begin{aligned}
& \dot{A}_{1}=\lambda_{1} A_{1}+A_{1}\left(a\left|A_{1}\right|^{2}+b\left(\theta_{\text {res }}\right)\left|A_{2}\right|^{2}\right)+\ldots \\
& \dot{A}_{2}=\lambda_{1} A_{2}+A_{2}\left(b\left(\theta_{\text {res }}\right)\left|A_{1}\right|^{2}+a\left|A_{2}\right|^{2}\right)+\ldots,
\end{aligned}
$$

where

$$
b\left(\theta_{\text {res }}\right)=b_{0}+b_{\text {res }}, \quad b_{\text {res }}=-\frac{\alpha_{1} \alpha_{2}}{\lambda_{2}} .
$$

The presence of the weakly damped mode therefore changes the value of the crosscoupling coefficient $b\left(\theta_{\text {res }}\right)$ between modes with wavevectors $\mathbf{k}_{\mathbf{1}}$ and $\mathbf{k}_{\mathbf{2}}$, offset at an angle $\theta_{\text {res }}$. The value of $\theta_{\text {res }}$ is determined by the ratio of critical and weakly damped 
wavenumbers, but the same idea holds for any three-wave interaction between any $\mathbf{k}_{\mathbf{1}}$ and $\mathbf{k}_{\mathbf{2}}$ on the critical circle. This results in a function $b(\theta)$ with either a distinctive peak or dip at $\theta=\theta_{\text {res }}$, depending on the sign of $\alpha_{1} \alpha_{2}$ and on the weakness of the damping for the weakly damped mode.

Analysing the amplitude equations $(4.3)$ shows that there are two types of solutions that bifurcate from the trivial solution $A_{1}=A_{2}=0$, namely stripes $\left(A_{1} \neq 0, A_{2}=0\right.$ or vice versa) and rectangles $\left(A_{1}=A_{2}\right)$. The relative stability of rectangles to stripe perturbations is dependent on the relative size of the self-coupling coefficient $a$ in equation (4.3) and $b\left(\theta_{\text {res }}\right)$ where, if $b_{\text {res }}>0\left(\alpha_{1} \alpha_{2}>0\right)$, then the stability of rectangles is enhanced by the three-wave interaction and if $b_{\text {res }}<0\left(\alpha_{1} \alpha_{2}<0\right)$, then the stability of rectangles is suppressed.

Of course, in the Faraday problem there are not just two modes and there are many interactions and several weakly damped circles, but this idea that three-wave resonances can promote patterns associated with the angle $\theta_{\text {res }}$ is powerful (Silber et al. (2000)). The idea is that the dispersion relation determines which wavenumbers are critical or close to critical; allowed three-wave resonant interactions then select out particular wavevectors; the allowed wavevectors are necessarily oriented at particular angles as determined by the ratios of available wavenumbers, leading to specific values for $\theta_{\text {res }}$. Superlattice patterns essentially consist of a nonlinear superposition of two set of hexagons offset at an angle to each other. There is a whole family of different superlattice patterns (Dionne et al. (1997)), each corresponding to a different angle, but the particular superlattice that will be promoted will be that with an angle given by $\theta_{\text {res }}$.

The superlattice patterns observed by Kudrolli et al. (1998) for $\{6,7\}$ excitation exemplify the idea. The superlattice patterns that they observe are spatially periodic and a spatial Fourier transform of the pattern indicates that they consist of essentially two sets of hexagonal modes offset by an angle of $22^{\circ}$. In this case, the particular three-wave resonance of relevance is of the type illustrated in figure 4(b) and is between a harmonic mode that is weakly damped of frequency $2 \omega / 2$, related to the $2 \omega / 2$ tongue, and two wavevectors with wavenumbers corresponding to the $6 \omega / 2$ tongue (Silber et al. $(2000)$ ). This was surprising because the superlattice state appears near the bicritical point where both $6 \omega / 2$ and $7 \omega / 2$ onset simultaneously and naively, from inspection of the linear stability diagram (see figure 2(b)) one would assume that it was a result of the interaction of $6 \omega / 2$ and $7 \omega / 2$ modes - the $2 \omega / 2$ tongue onsets at an amplitude of approximately 50 so is not even visible on the scale shown.

This argument not only explains the presence of the $22^{\circ}$ superlattice patterns but also why patterns with angles close to $30^{\circ}$ are seen in $j=\{4,5\}$ forcing (Skeldon \& Guidoboni (2007)). Extensions of this basic idea have been used to suggest ways to design forcing frequencies to promote particular patterns in both Faraday waves (Porter et al. (2004)) and in a model partial differential equation (Rucklidge \& Silber (2009)).

The results of Kudrolli et al. (1998) and the theoretical results of Silber et al. (2000) showed how modes visible in the spatial Fourier transform of the experimentally observed pattern can be linked to critical/weakly damped modes and three-wave resonant interactions. It follows that given an experimentally observed pattern it should be possible to confirm whether or not it is a result of a particular interaction by establishing which modes are present in the pattern. However, identifying precisely which modes are involved can be tricky, as we illustrate in the following section.

\subsection{Practical identification of relevent modes}

In Epstein \& Fineberg (2006) a detailed comparison of different patterns, referred to as 'grid' states and labelled as $3: 2,4: 3$ and $5: 3$ respectively is presented. The spatial 
(a)

(c)
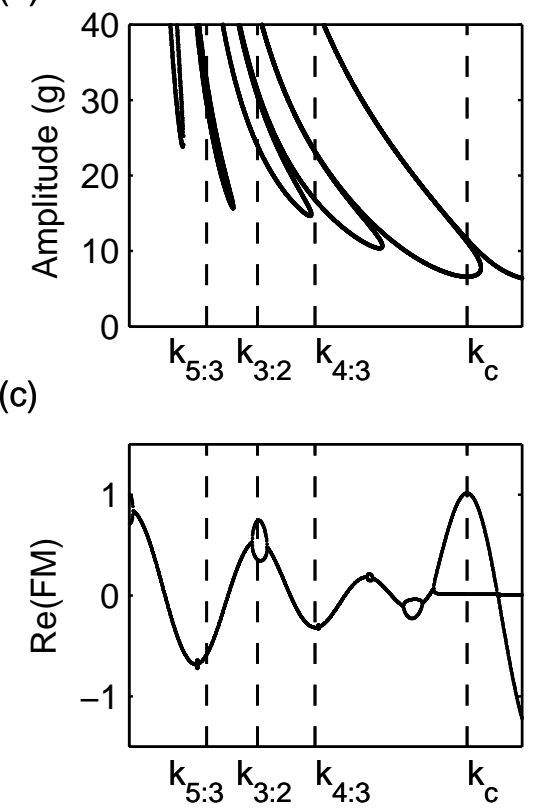

(b)

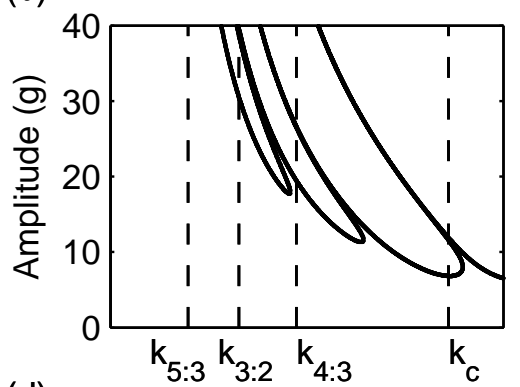

(d)

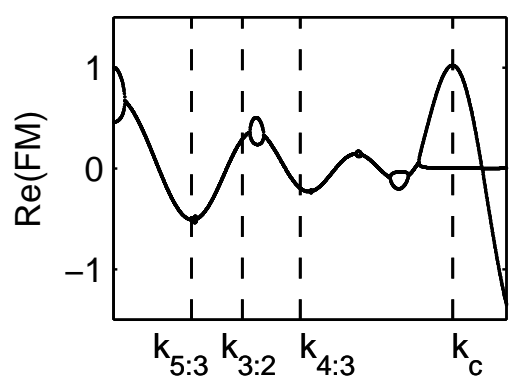

Figure 5. Linear stability curves and Floquet multipliers calculated from the finite depth Navier-Stokes equations (a) and (c) $h=0.3 \mathrm{~cm}$ (b) and (d) $h=0.2 \mathrm{~cm}$. Parameter values are taken from Epstein \& Fineberg (2006) and are $\sigma=20.6 \mathrm{dyn}^{-1} ; \nu=0.18 \mathrm{~cm}^{2} \mathrm{~s}^{-1}$; $\rho=0.949 \mathrm{~g} \mathrm{~m}^{-3} ; a_{j}, j=\{6,7,2\}$, where $a_{6}=3.4, a_{7}=6.4, a_{2}=0.5 ; \phi_{j}=0^{\circ} ; \omega / 2 \pi=14 \mathrm{~Hz}$.

Fourier transform of the patterns enable the identification of the wavenumbers that are present in these patterns: in each case they appear to be dominated by modes sitting on two different circles, one on the primary harmonic instability with wavenumber $k_{c}$ and a second circle with respective wavenumbers $k_{3: 2}=0.38 k_{c}, k_{4: 3}=0.55 k_{c}$ and $k_{5: 3}=0.23 k_{c}$. These ratios are fixed by the fact that the observed grid states consist of modes that fit on to a hexagonal lattice. This suggests that they are strong candidates for superlattice patterns that occur as a result of the mechanism discussed in section 4.1. Epstein \& Fineberg (2006) computed the linear stability curves, and their linear stability diagrams are re-computed in figure 5(a) and (b). As stated by Epstein \& Fineberg (2006) and, as can be seen in this figure, the $k_{3: 2}$ wave number lines up with the first harmonic tongue $(2 \omega / 2)$ and this strongly suggests that it is the same mechanism that produces the familiar $22^{\circ}$ superlattice patterns, seen by Kudrolli et al. (1998), that produces the $k_{3: 2}$ grid state. For the two other states the situation is less clear. Epstein \& Fineberg (2006) state that $k_{4: 3}$ lines up with the the second harmonic tongue $(4 \omega / 2)$, but this is not clear from figure 5 (the position of the line drawn in figure 5 of Epstein \& Fineberg (2006) is not consistent with the value of $0.55 k_{c}$ given). Epstein \& Fineberg (2006) observe that the $k_{5: 3}$ mode does not appear to be aligned with any tongue and that the $5: 3$ pattern occurs in a wedge-shaped region of parameter space that emerges from the bicritical point, unlike the $3: 2$ and the $4: 3$ state that occur at a short distance from this point.

The difficulty here is that the linear stability diagram indicates that at an amplitude of excitation of approximately $7.5 \mathrm{~g}$ the mode with wavenumber $k_{c}$ and frequency $6 \omega / 2$ becomes unstable and that there are five other damped modes with smaller wavenumber. But the position of the tongues for these modes only give a rough idea of the precise 
wavenumber and damping associated with each mode at the pattern onset amplitude of $7.5 \mathrm{~g}$.

More accurate information on the damped modes can be obtained by calculating the most critical Floquet multipliers, as detailed in Appendix A The results for the parameter values used by Epstein \& Fineberg (2006) are shown in figure 5(c) and (d). As identified by Epstein \& Fineberg (2006), the $k_{3: 2}$ mode is aligned with the harmonic tongue associated with frequency $2 \omega / 2$ for $h=0.3 \mathrm{~cm}$, but not for $h=0.2 \mathrm{~cm}$ and supports their observation that the $k_{3: 2}$ grid state will be seen for $h=0.3 \mathrm{~cm}$ and not for $h=0.2 \mathrm{~cm}$. The remaining two wavenumbers $k_{5: 3}$ and $k_{4: 3}$ are very close to the subharmonic waves of frequencies $\omega / 2$ and $3 \omega / 2$ respectively. This clarifies the issue that the $k_{5: 3}$ mode in Epstein \& Fineberg (2006) did not appear to align with any mode on the linear stability graphs and strongly suggests that the $k_{4: 3}$ mode was mis-identified as harmonic. However, it also presents a problem in that temporal constraints mean that it is not possible for two harmonic wavevectors to form a three-wave resonance with a single subharmonic wavevector. Consequently, $\alpha_{1}=\alpha=0$ in equations $(4.2)$, so the discussion in section 4.1 does not hold as it stands and there is no special angle $\theta_{\text {res }}$.

Epstein \& Fineberg (2006) point out that the $k_{5: 3}$ case could be a result of a threewave resonance between modes with frequencies $\omega / 2,6 \omega / 2$ and $7 \omega / 2$ and this is strongly supported by our Floquet multiplier calculation and is a permitted resonance in that it satisfies both spatial and temporal constraints. Such a resonance appears to be consistent with the spatial Fourier transform in figure 3(c) of Epstein \& Fineberg (2006) and is similar in structure to previous states identified in figure 20(c) of Arbell \& Fineberg (2002) as 2MS states. This would make it a three-wave resonance of the form shown in figure 4 (d). This kind of resonance was discussed briefly by Porter \& Silber (2002) and results in patterns that occur in a wedge that emerges from the bicritical point. This is consistent with the bifurcation sets shown by Epstein and Fineberg. The only remaining conundrum for this particular pattern is that Epstein and Fineberg state that, although all the evidence points to subharmonic modes being important, no subharmonic component was found.

\section{Observed patterns near onset: theoretical bifurcation sets compared with experiment}

In spite of the difficulties in identifying modes, nevertheless, in experiments with dominant forcing frequencies $j=\{4,5\}$ or $j=\{6,7\}$ there is a coherent picture: in the $\{6,7\}$ case, observed superlattice patterns near onset have an angle of $22^{\circ}$; for $j=\{4,5\}$, observed patterns near onset are quasipatterns with an angle of $30^{\circ}$. Although there are technical difficulties with considering quasipatterns in the same way as superlattice patterns (Rucklidge \& Rucklidge (2003); Rucklidge \& Silber (2009); Iooss \& Rucklidge (2010)), nevertheless they appear to fit within the same framework with resonant interactions with weakly damped modes associated with $2 \omega / 2$ contributing to $b_{\text {res }}$ and explaining the appearance of the appropriate angle. The different angle seen in the $\{4,5\}$

case as compared with the $\{6,7\}$ case is because of differences in the wavenumbers of the interacting modes. In both cases, superlattice/quasipatterns are only seen near the bicritical point where the two modes driven by the two main components of the forcing onset simultaneously.

Here, we aim to carry out a careful comparison of the results of weakly nonlinear theory and experimental results to explore to what extent there is quantitative agreement between experiments and theory as the bicritical point is approached. We present results from the experiments of Ding \& Umbanhowar (2006) because we have excellent agree- 
ment for the linear stability curves with $\nu=0.21 \mathrm{~cm}^{2} \mathrm{~s}^{-1}$, as shown in figure 3(e) and (f), and this set of experiments includes the most comprehensive study of how multiple frequencies interact via the superlattice mechanism outlined above. Specifically, acknowledging the importance of the $2 \omega / 2$ tongue, Ding \& Umbanhowar (2006) carry out a series of experiments that systematically explore the effect of adding a third frequency that promotes this mode.

The weakly nonlinear theory is carried out using the method given in Skeldon \& Guidoboni (2007) for analysing equations (2.6) to (2.7). For all details of how weakly nonlinear analysis is used to derive coefficients for the relevant amplitude equations we refer the reader to Skeldon \& Guidoboni (2007). In order to examine the relative stability of superlattice patterns a minimal set of twelve amplitude equations are needed. However, the stability of the planforms that bifurcate from the non-patterned state can be found by calculating the coefficients on three one-dimensional subspaces of this twelve dimensional problem: one describing stripes, one for hexagons and one for rectangles generated from wavevectors separated by an angle $\theta$ as detailed in Skeldon \& Silber (1998). By varying $\theta$, this formulation then results in determining the stability of stripes to any perturbation of the same wavenumber; the stability of hexagons to (i) stripes arbitrarily close to any given orientation (ii) rectangles arbitrarily close to any given aspect ratio (iii) to superlattice patterns of any angle; the stability of rectangles to (i) stripes of any orientation (ii) superlattice patterns on the same lattice; the stability of superlattice patterns to hexagons, stripes or rectangles that are made up of a subset of the superlattice wavevectors.

Since the weakly nonlinear stability analysis indicates that for many parameter values there is more than one stable pattern, results are presented in two ways, first showing the regions of stability for individual patterns - only those that have some region of stability are shown, and secondly showing the bifurcation set for the most stable state, as computed from the weakly nonlinear coefficients using the Lyapunov functional given in Skeldon \& Guidoboni (2007). The most stable states are superimposed on the experimental results of Ding \& Umbanhowar (2006). In the case of superlattice patterns, the resonant interactions mean that it is always the particular pattern associated with $\theta_{\text {res }}$ that has the largest region of stability and it is perturbations associated with this angle that first destabilise hexagons. Consequently where superlattice patterns are shown it is always the superlattice pattern associated with $\theta_{\text {res }}$ that is relevant.

Ding and Umbanhowar consider the two sets of frequency components most widely used by other experimental groups, namely $j=\{4,5\}$ and $j=\{6,7\}$ and then systematically investigate the inclusion of a third mode, so considering the combinations $j=\{4,5,2\}$ and $j=\{6,7,2\}$. We have carried out the weakly nonlinear stability analysis for each of their parameter studies and the results are presented in figures 7.12 where the principle parameters considered and the corresponding figures in Ding \& Umbanhowar (2006) are summarised in table 1.

In each case the weakly nonlinear results are superimposed on the experimental results. The parameters have been chosen to match those quoted in the paper of Ding \& Umbanhowar (2006) with the exception of the viscosity where, as seen in figure 3 , the linear stability curves fitted best with a viscosity of $\nu=0.21 \mathrm{~cm}^{2} \mathrm{~s}^{-1}$ rather than $\nu=0.20$ $\mathrm{cm}^{2} \mathrm{~s}^{-1}$ quoted in their paper (or the value of $\nu=0.204 \mathrm{~cm}^{2} \mathrm{~s}^{-1}$ quoted in Ding (2006)).

\subsection{Results for $\{4,5\}$ and $\{4,5,2\}$}

In figure 6 we see that the weakly nonlinear theory predicts bistability between squares and hexagons for low values of $a_{5}$, with squares losing stability as the bicritical point is approached. There is good agreement between the point at which squares become 

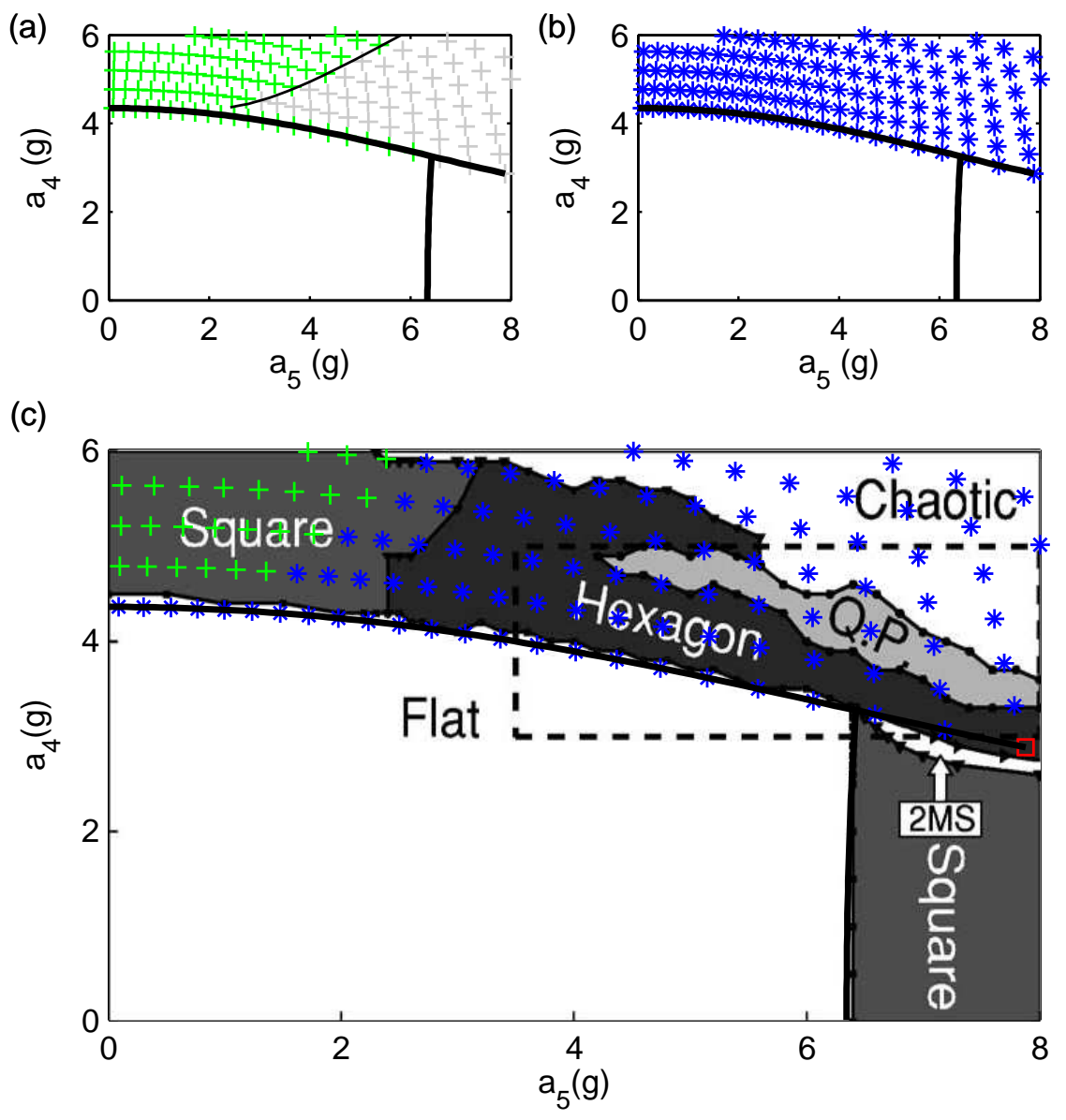

Figure 6. Comparison with figure 1 from Ding \& Umbanhowar (2006). $a_{j}=\{4,5\},\left(\phi_{4}, \phi_{5}\right)=\left(0,16^{\circ}\right), \omega / 2 \pi=20 \mathrm{~Hz}$. The fluid parameters are as in the caption to figure 3(e),(f) with $\nu=0.21 \mathrm{~cm}^{2} \mathrm{~s}^{-1}$. (a)-(b) Stability regions for different planforms. (a) Stable squares in green; unstable squares in grey; (b) stable hexagons in blue; unstable hexagons in grey. (c) 'Most stable' state using a Lyapunov energy argument: + squares; * hexagons; $\square$ quasipatterns. The theoretical results are overlaid on figure 1 from Ding \& Umbanhowar (2006). Adapted with permission from Ding \& Umbanhowar (2006). Copyrighted by the American Physical Society.

\begin{tabular}{|c|c|c|c|c|}
\hline Figure & $j$ & $a_{j}, \phi_{j}$ & $\begin{array}{c}\text { Parameters } \\
\text { varied }\end{array}$ & $\begin{array}{c}\text { Figure from } \\
\text { Ding \& Umbanhowar }\end{array}$ \\
\hline 6 & $\{4,5\}$ & $\left(\phi_{4}, \phi_{5}\right)=\left(0,16^{\circ}\right)$ & $a_{4}$ and $a_{5}$ & 1 \\
\hline 7 & $\{4,5,2\}$ & $a_{2}=0.8 g,\left(\phi_{4}, \phi_{5}, \phi_{2}\right)=\left(0^{\circ}, 16^{\circ}, 32^{\circ}\right)$ & $a_{4}$ and $a_{5}$ & $3(b)$ \\
\hline 8 & $\{4,5,2\}$ & $a_{5}=5 g,\left(\phi_{4}, \phi_{5}, \phi_{2}\right)=\left(0^{\circ}, 5^{\circ}, 32^{\circ}\right)$ & $a_{4}$ and $a_{2}$ & $4(\mathrm{a})$ \\
\hline 9 & $\{4,5,2\}$ & $\left(a_{4}, a_{5}, a_{2}\right)=(3.8,5.8,0.8) g, \phi_{4}=0^{\circ}$ & $\phi_{5}$ and $\phi_{2}$ & $4(\mathrm{~b})$ \\
\hline 10 & $\{6,7$ & $\left(\phi_{6}, \phi_{7}\right)=\left(0^{\circ}, 40^{\circ}\right)$ & $a_{6}$ and $a_{7}$ & 2 \\
\hline 111 & $\{6,7,2\}$ & $a_{7}=7 g,\left(\phi_{6}, \phi_{7}, \phi_{2}\right)=\left(0^{\circ}, 40^{\circ}, 80^{\circ}\right)$ & $a_{6}$ and $a_{2}$ & $6(\mathrm{a})$ \\
\hline 12 & $\{6,7,2\}$ & $\left(a_{6}, a_{7}, a_{2}\right)=(5.2,7.8,0.6) g, \phi_{6}=0^{\circ}$ & $\phi_{7}$ and $\phi_{2}$ & $6(\mathrm{~b})$ \\
\hline
\end{tabular}

TABLE 1. Summary information for figures comparing weakly nonlinear analysis with the experiments of Ding \& Umbanhowar (2006). 
unstable (approximately $a_{5}=2.2 g$, figure 6(a)), and the transition between squares and hexagons in the experiment. However, before squares become unstable, the theory suggests that there is a region of bistability between hexagons and squares and that in this region hexagons and not squares, as seen in the experiment, are the most stable state. Of course, in this region, the particular pattern observed experimentally will depend to some extent on the way in which the experiments were carried out. For example, if the experimental procedure was to fix $a_{4}$ and to increase $a_{5}$ from zero in small steps, one would expect to see squares until the limit of their stability. Regions of quasipatterns are also predicted by the weakly nonlinear theory (almost off the scale of the figure 6) and observed in the experiment but since they do not occur close to onset it is perhaps not surprising that the theory and experiment do not agree.

In figure 7, we see that by adding in a forcing component that excites the $2 \omega / 2$ mode directly Ding \& Umbanhowar (2006) showed that they could increase the region of quasipatterns, to the extent that they become the first pattern observed after the unpatterned state becomes unstable. Our theoretical results for the same parameter values also show an enhanced region for the superlattice patterns - but not as enhanced as for the experiment. Two possible reasons for this are (i) the nearby presence of the bicritical point where both the $2 \omega / 2$ and the $4 \omega / 2$ tongue onset simultaneously which means that our weakly nonlinear calculations would need to be extended; (ii) the sensitivity of the results to phase. We discuss both of these in more detail below.

Figure 8 shows excellent agreement between the theoretical results and the experimental results for the pattern at onset for increasing $a_{2}$, with agreement diminishing with distance from onset.

There is surprisingly good agreement for the variation with phase shown in figure 9 . In the experimental results, black indicates low correlation at an angle of $30^{\circ}$. This lines up well with areas where the theory indicates competition between squares and hexagons. White areas, where there is high correlation at $30^{\circ}$, line up well with stable quasipatterns. In the regions where there are no coloured symbols, the weakly nonlinear analysis indicates no stable states. This region is mostly mid-grey in the experiment, indicating some correlation at $30^{\circ}$.

The agreement in figure 9 is surprising because the results correspond to a point with $a_{4}=3.8 g, a_{5}=5.9 g$ and $a_{2}=0.8 g$, which according to the theory is close to a transition from quasipatterns to hexagons in a region where both hexagons and quasipatterns are stable, whereas in the experiment it is firmly in the quasipattern region. (In order to help cross-reference between the two figures, a black cross has been marked on both figure 7(c) and figure 9.) Figure 9 highlights the sensitivity of the results to phase and in fact, with our weakly nonlinear analysis we find that the size of the region of quasipatterns changes significantly depending on the phase. This sensitivity to the precise value of the phase could be part of the difficulty in obtaining agreement for the onset of quasipatterns in figure 7 .

\subsection{Results for $\{6,7\}$ and $\{6,7,2\}$}

In figure 10 the weakly nonlinear theory predicts bistability between squares and hexagons for low values of $a_{7}$, with squares losing stability as the bicritical point is approached. As for figure 6, although there is good agreement between the point at which squares become unstable (approximately $a_{7}=4.5 \mathrm{~g}$ ), and the transition between squares and hexagons in the experiment, this agrees less well with the results of the energy argument.

In figure 11 both theory and experiment agree that for low and moderate values of $a_{2}$ hexagons are the preferred pattern. As $a_{2}$ increases, hexagons are replaced by superlattice patterns, although the transition observed experimentally occurs slightly earlier than in 

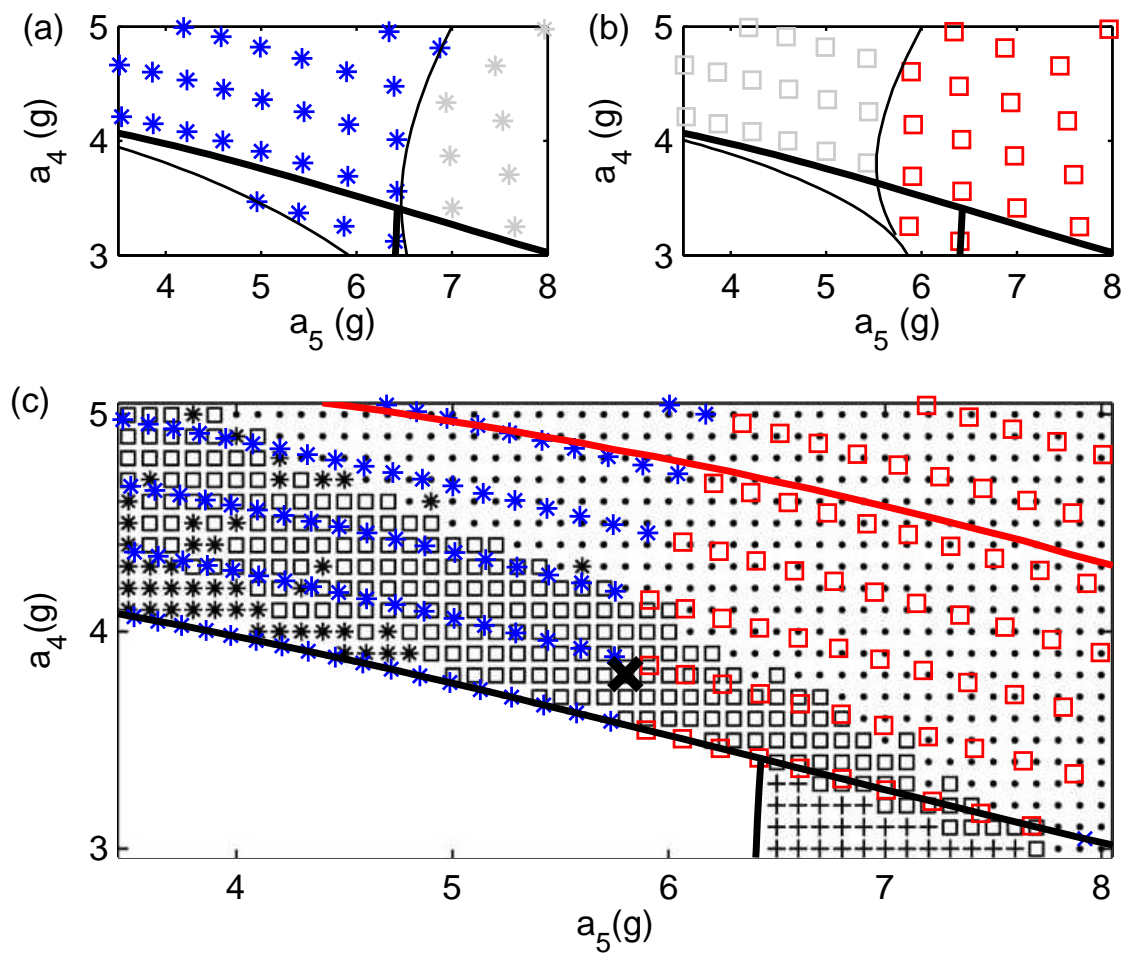

Figure 7. Comparison with Ding \& Umbanhowar (2006) Figure 3(b), $j=\{4,5,2\},\left(\phi_{4}, \phi_{5}, \phi_{2}\right)=\left(0,16^{\circ}, 32^{\circ}\right)$. (a)-(b) Stability regions for different planforms. (a) Stable hexagons in blue, unstable hexagons in grey; (b) stable quasipatterns in red, unstable quasipatterns in grey. (c) 'Most stable' planform using a Lyapunov energy argument superimposed on figure 3(b) from Ding \& Umbanhowar (2006). Adapted with permission from Ding \& Umbanhowar (2006). Copyrighted by the American Physical Society. $*$ hexagons; $\square$ quasipatterns, $\bullet$ disordered states, where the coloured symbols are the theoretical results and the black symbols the experimental results. The red line is the linear instability curve for the harmonic $2 \omega / 2$ tongue. The black cross indicates the point at which the experiment shown in figure 9(c) was carried out.

the weakly nonlinear calculations. Away from onset, the agreement is qualitative rather than quantitative: in the experiments superlattice patterns are observed for a much larger region than predicted by the weakly nonlinear theory and hexagons are observed in regions where the theory predicts bistability of rectangles and hexagons, with rectangles being the most stable state. For large values of $a_{2}$ and $a_{6}$, the experiments see disordered states. The weakly nonlinear theory cannot predict such states, but note that none of the planforms considered are found to be stable for large $a_{2}$ and $a_{6}$ (there are no symbols from the theoretical calculations in the top right of figure 11.

The results shown in figure 12 again show that the theoretical results have the same diagonal dependency as seen in the experiments, a consequence of a phase invariant as discussed further below. For the experimental results, white indicates a high correlation with the angle of $22^{\circ}$ and this does seem to align with where superlattice patterns occur, although for much of the lighter regions the weakly nonlinear analysis predicts no stable pattern. The black regions correspond to a low correlation at $22^{\circ}$ and Ding \& Umbanhowar (2006) state that this region contains 'disordered' patterns: our theoretical results suggest that both rectangles and hexagons are stable for much of this region each having 

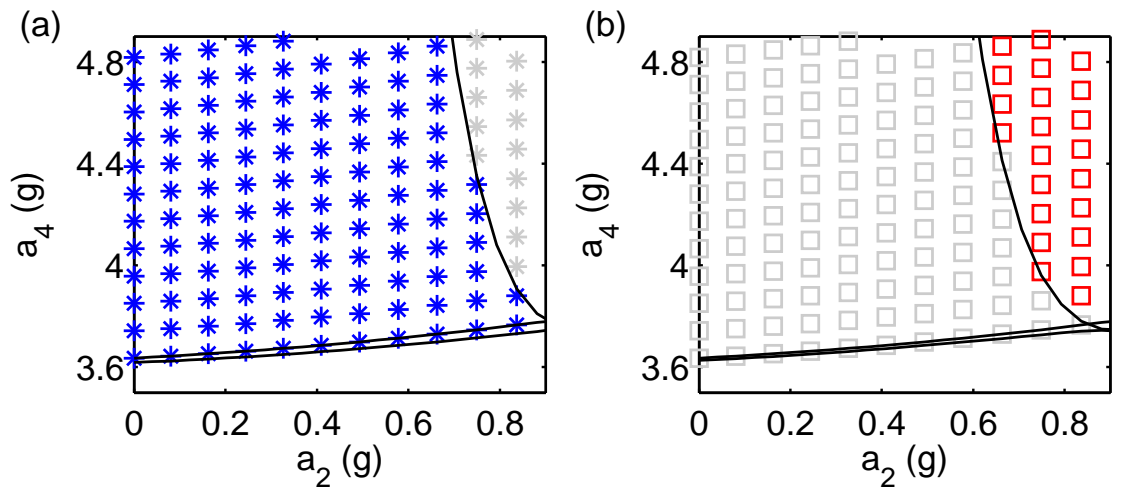

(c)

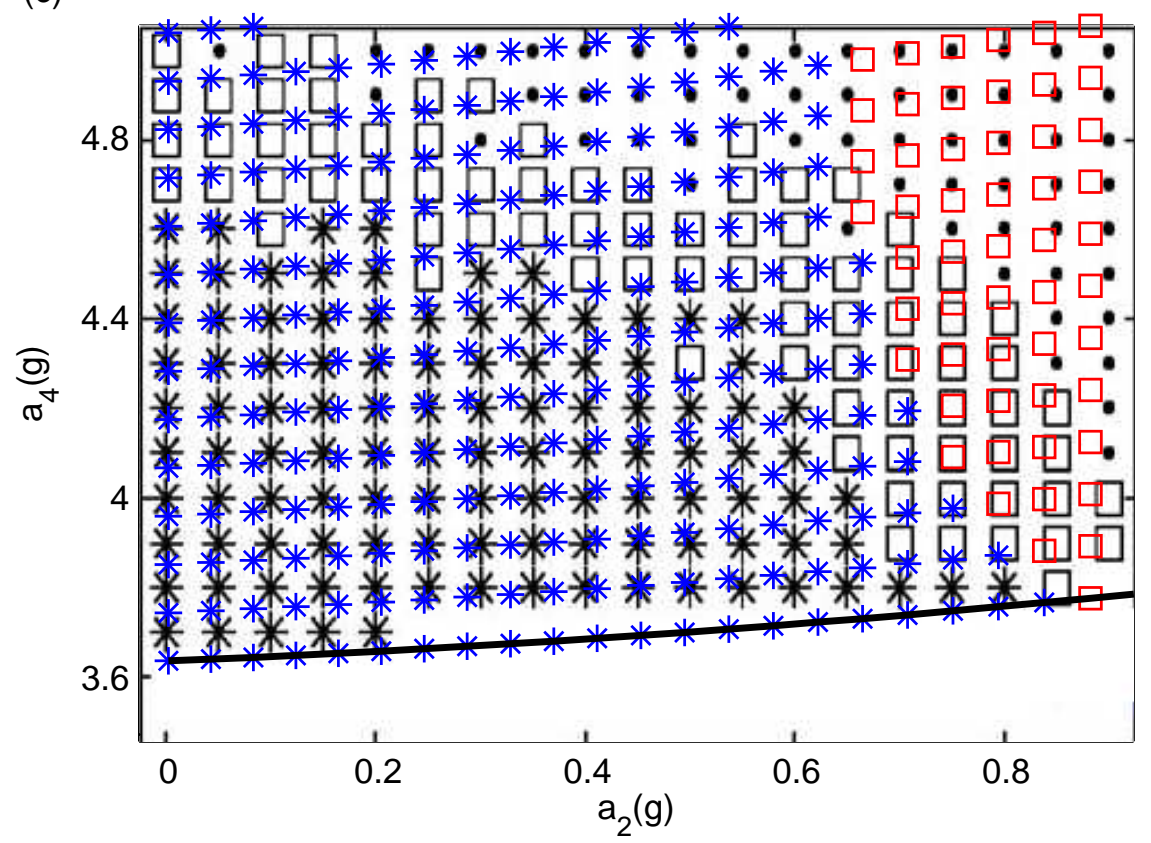

Figure 8. Comparison with Ding \& Umbanhowar (2006) figure 4(a). $j=\{4,5,2\}, a_{5}=5 g,\left(\phi_{4}, \phi_{5}, \phi_{2}\right)=\left(0,5^{\circ}, 32^{\circ}\right)$. (a) and (b) stability regions for hexagons and quasipatterns respectively. Coloured symbols stable; grey unstable. Hexagons and quasipatterns both bifurcate from the unpatterned state at a transcritical bifurcation, where the unstable bifurcating branch changes direction at a saddle-node bifurcation. This leads to regions of stable hexagons and quasipatterns that are subcritical. (c) 'Most stable' planform: * hexagons; $\square$ quasipatterns, overlaid on figure 4(a) from Ding \& Umbanhowar (2006). Adapted with permission from Ding \& Umbanhowar (2006). Copyrighted by the American Physical Society. The coloured symbols are the theoretical results and the black symbols the experimental results. The black dots indicate disordered states. Note that the linear stability boundary for the $2 \omega / 2$ mode is just off this diagram, and is approximately parallel to the $a_{4}$ axis through a value of $a_{2} \approx 1$. The bicritical point where the $2 \omega / 2$ and the $4 \omega / 2$ modes onset simultaneously occurs when $\left(a_{2}, a_{4}\right)=(1.05,3.80) \mathrm{g}$.

a similar Lyapunov energy. Consequently one might expect competition between these two states, resulting in the observed disorder. 
(a)

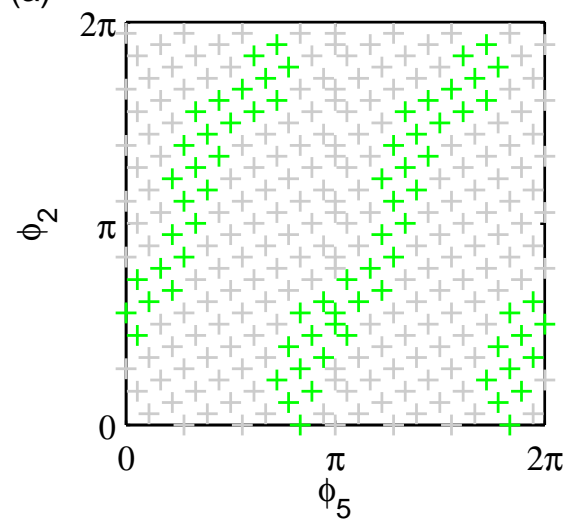

(c)

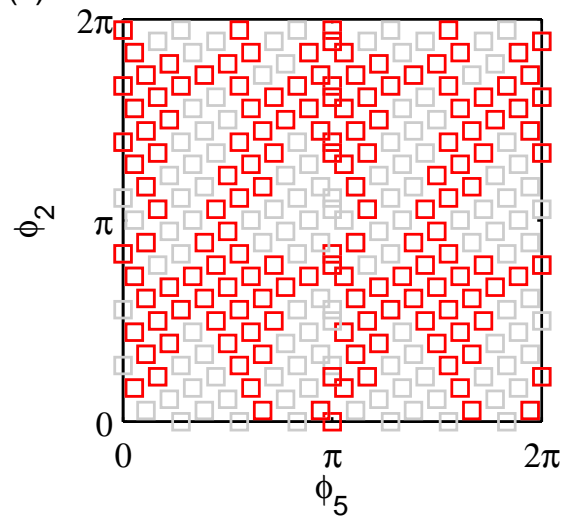

(b)

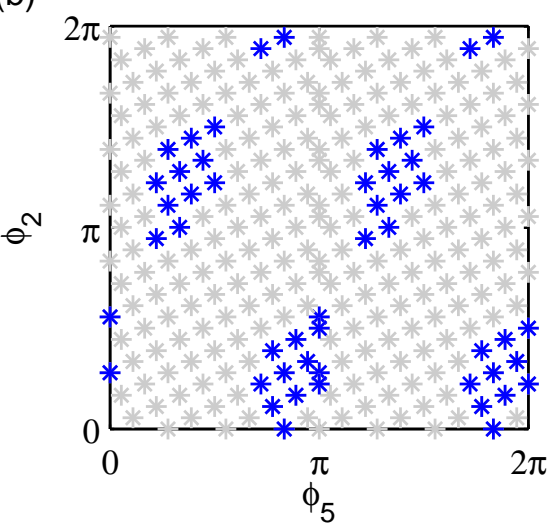

(d)

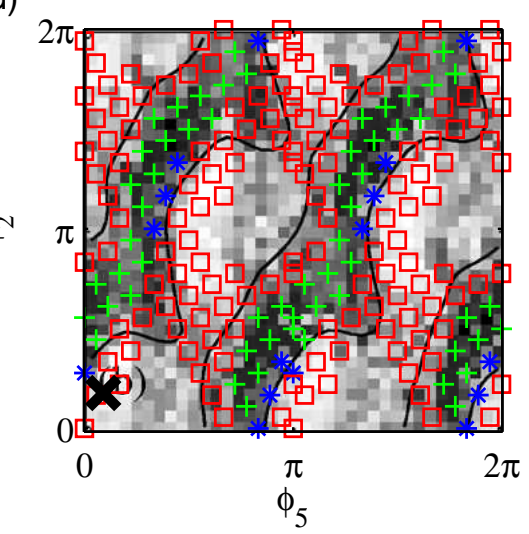

Figure 9. Comparison with Ding \& Umbanhowar (2006) figure 4(b). Phase diagram for $j=\{4,5,2\}$ with $\left(a_{4}, a_{5}, a_{2}\right)=(3.8,5.8,0.8) g$. (The position of this point for $\left(\phi_{5}, \phi_{2}\right)=\left(16^{\circ}, 32^{\circ}\right)$ is shown in figure 7 (c).) (a)-(c) stability regions for squares, hexagons and quasipatterns respectively. Coloured symbols stable; grey unstable. (d) 'Most stable' planforms: + squares; * hexagons; $\square$ quasipatterns. The results are overlaid on figure 4(b) of Ding \& Umbanhowar (2006). Adapted with permission from Ding \& Umbanhowar (2006). Copyrighted by the American Physical Society. The cross indicates the point which corresponds to the cross in figure 7

\subsection{Common themes}

From our comparison, a number of themes emerge:

(a) There is excellent agreement with theory at onset in some cases (figure 8).

(b) In other cases, figure 6 and 10 bistability of patterns makes it difficult to compare theory and experiment. The experimental results for the pattern at onset are consistent with the theory, but sometimes the pattern at onset is not that predicted by considering the Lyapunov argument. This is one difficulty in comparing with experiment post festum, since in regions of bistability, which pattern is observed can depend on the way that the experiments are performed.

(c) When the additional forcing component $\left(a_{2} \neq 0\right)$ is included that promotes the mode with frequency $2 \omega / 2$, then in both theory and experiments patterns associated with the resonant angle are promoted and consequently appear for a larger region of parameter space. 
(a)

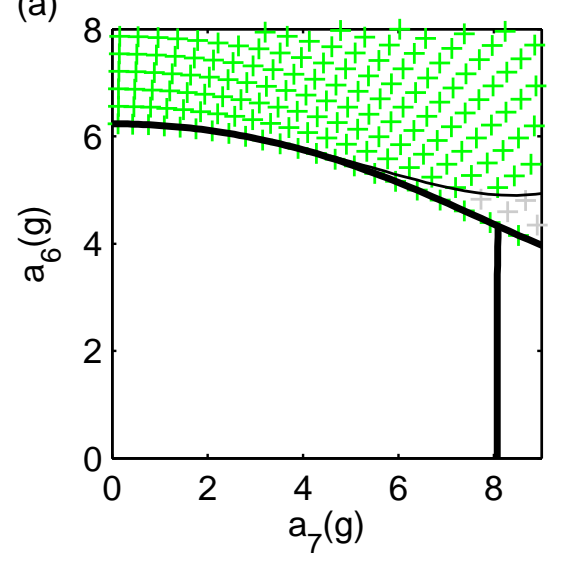

(b)
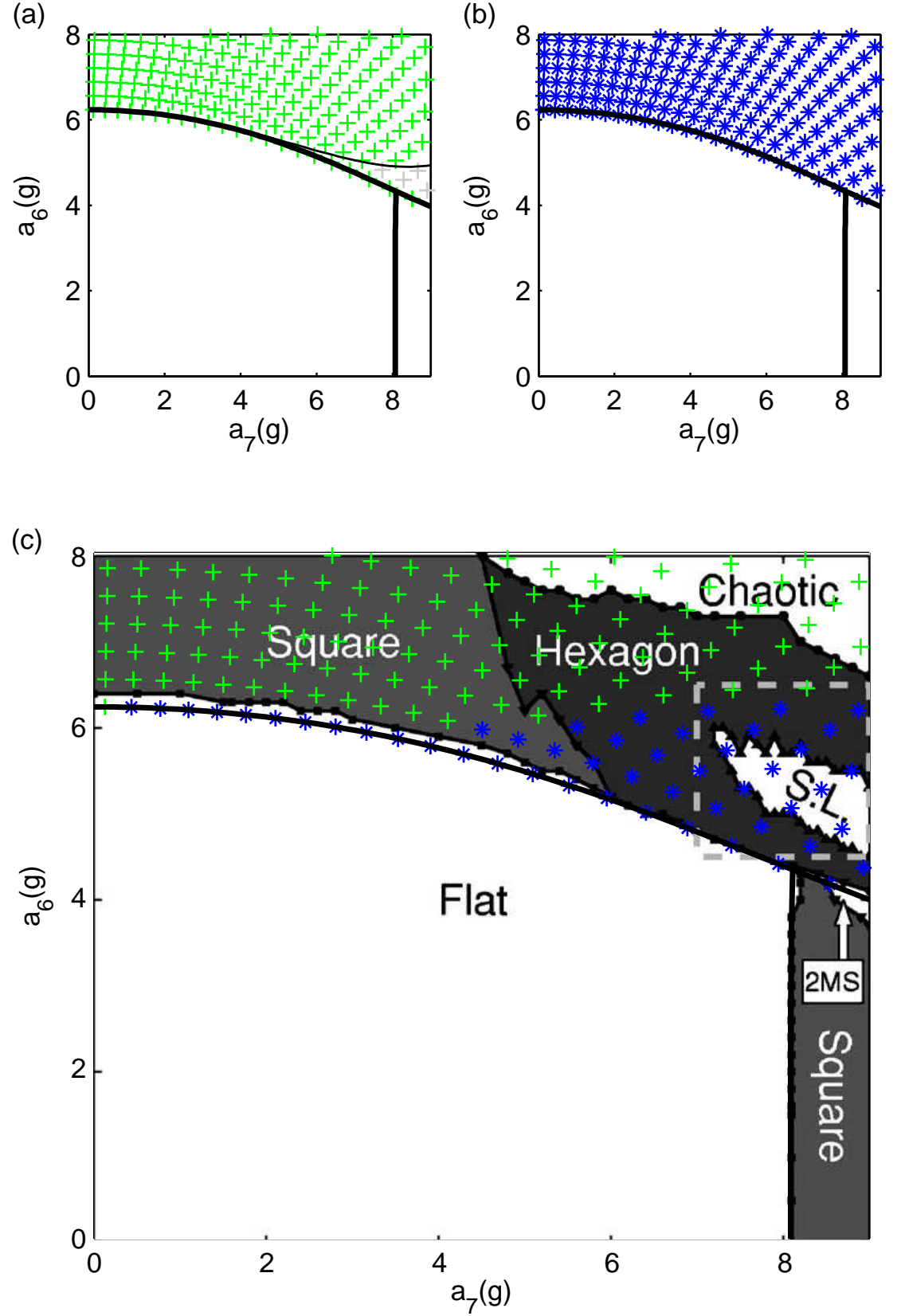

Figure 10. Comparison with Ding \& $\quad$ Umbanhowar (2006), figure 2. $j=\{6,7\},\left(\phi_{6}, \phi_{7}\right)=\left(0,40^{\circ}\right), \omega / 2 \pi=16.5 \mathrm{~Hz}$. (a)-(b) stability regions for rectangles and hexagons. Coloured symbols stable; grey unstable. (c) 'Most stable' planform: + rectangles; * hexagons, overlaid on figure 2 of Ding \& Umbanhowar (2006). Adapted with permission from Ding \& Umbanhowar (2006). Copyrighted by the American Physical Society. 

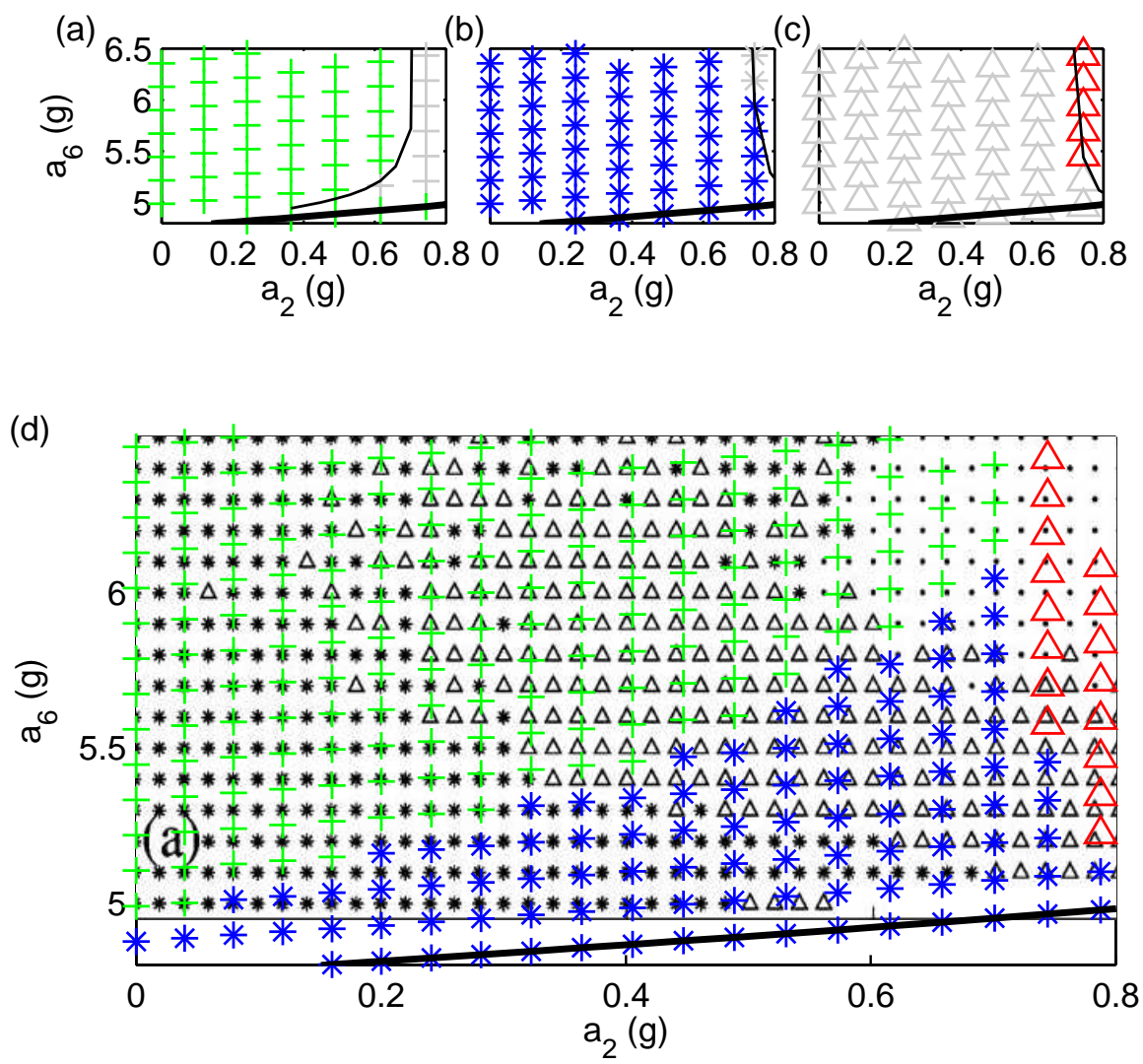

Figure 11. Comparison with Ding \& Umbanhowar (2006), figure 6(a). $j=\{6,7,2\}, a_{7}=7 g,\left(\phi_{6}, \phi_{7}, \phi_{2}\right)=\left(0,40^{\circ}, 80^{\circ}\right), \omega / 2 \pi=16.5 \mathrm{~Hz}$. (a)-(c) stability regions for rectangles, hexagons and superlattice patterns respectively. Coloured symbols stable; grey unstable. (d) 'Most stable' planforms superimposed on figure 6(a) from Ding \& Umbanhowar (2006). Adapted with permission from Ding \& Umbanhowar (2006). Copyrighted by the American Physical Society. The same symbol styles are used for both experiments and theory ( $\triangle$ superlattice; $*$ hexagons; + rectangles; $\bullet$ disordered states). Coloured symbols represent theoretical results and black symbols are the experimental results.

(d) In the experimental results, the additional forcing causes regions where both hexagonal states and superlattice patterns occur close to onset. This is also true in the weakly nonlinear analysis, but to a lesser extent, see figure 7 and 11 . There are two possible causes here: firstly, the theoretical results are sensitive to the phase, so changing the phase can expand the region of stable superlattice patterns. Secondly, the presence of the weakly damped $2 \omega / 2$ mode means that the results are in a region that is close to a bicritical point where both $2 \omega / 2$ and $4 \omega / 2$ modes onset simultaneously. In the neighbourhood of this bicritical point, one would expect the regions of stability as predicted from a codimension one analysis, at best, be perturbed, and, at worst, be inaccurate. We will return to this point in the discussion and note for now the red line in figure 7 that marks the onset of this mode.

(e) Quantitative agreement in most cases only occurs close to onset.

In addition, in all cases, close to the subharmonic instability boundary the theory predicts squares (not shown in the figures), as seen in the experiments. 
(a)

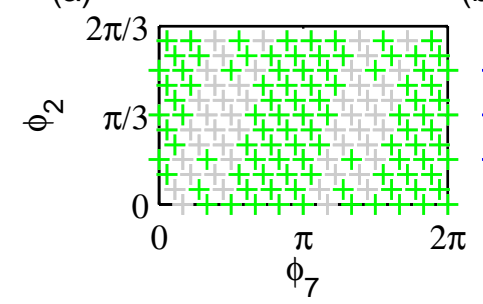

(b)

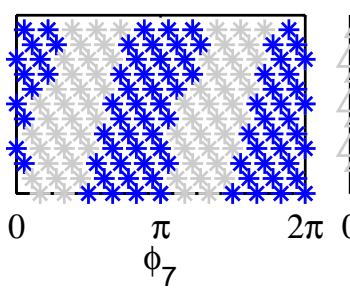

(c)

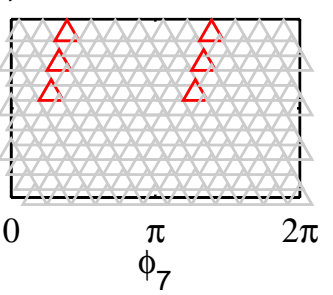

(d)

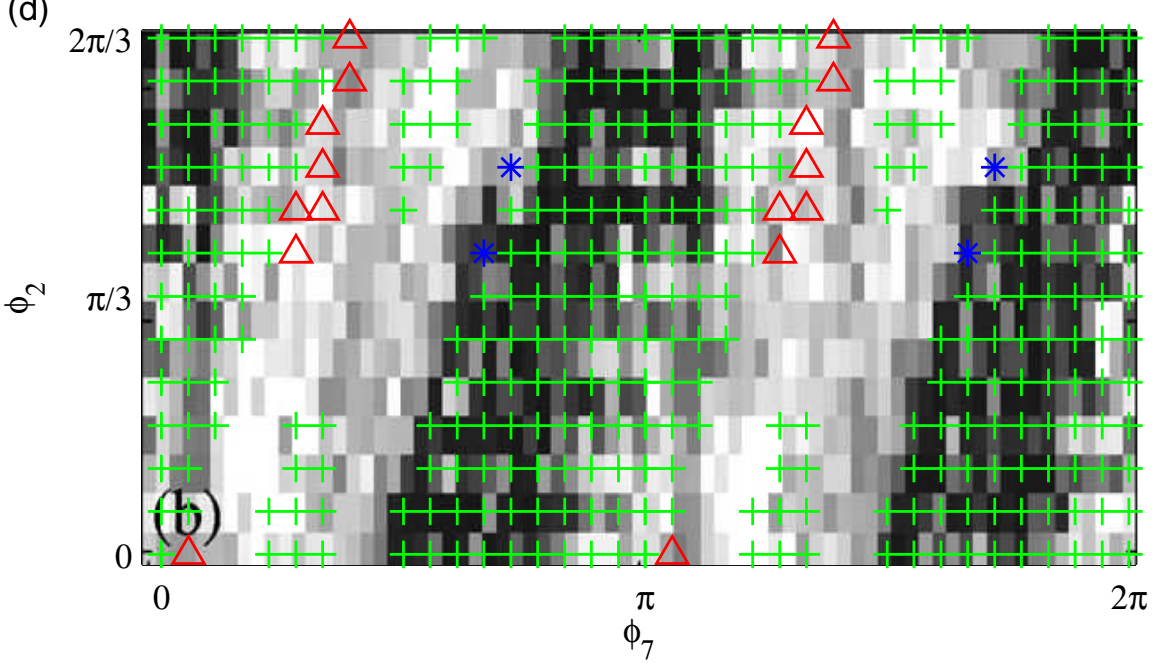

Figure 12. Comparison with Ding \& Umbanhowar (2006), figure 6(b). Phase diagram for $j=\{6,7,2\},\left(a_{6}, a_{7}, a_{2}\right)=(5.2,7.8,0.6) g, \omega / 2 \pi=16.5 \mathrm{~Hz}$. (a)-(c) stability regions for rectangles, hexagons and superlattice respectively. Coloured symbols stable; grey unstable. (d) 'Most stable' planform, superimposed on figure 6(b), Ding \& Umbanhowar (2006). $\triangle$ superlattice; $*$ hexagons; + rectangles. Coloured symbols represent theoretical Note that in (d) hexagons are rarely the 'most stable' planform: whenever hexagons are stable, rectangles usually are too. Although the Lyapunov energy for hexagons is often similar to that for rectangles, rectangles nearly always have the lowest value.

\section{Discussion}

The Faraday problem is an important pattern-forming system, yet it is particularly challenging to tie theory to experiment. The experiments are difficult to perform; the parameter regime of interest (large box, moderate viscosity) along with the technical difficulties of solving the free boundary Navier-Stokes equations make numerical solution of the problem hard, to the extent that although there has been some progress (see Périnet et al. (2012)) it is only very recently that any fully three-dimensional calculations of superlattice patterns in the Faraday problem have been reported Kahouadji et al. (2015). The fact that the instabilities result in an entire circle of unstable wavenumbers presents considerable theoretical difficulties (see Melbourne (1999)) and has meant that theory has, by necessity, restricted to a finite number of modes - the finite number normally chosen as the minimal set which will allow for the existence of the pattern of most interest. Furthermore, by its nature, weakly nonlinear theory cannot be expected to capture behaviour that is inherently strongly nonlinear: so a weakly nonlinear analysis 
of the transition from non-patterned to patterned state can only be expected to agree with experiment close to onset.

Nevertheless, our detailed comparison with Ding \& Umbanhowar (2006) shows very good agreement between experiment and weakly nonlinear results at onset, with the caveat that the theory often predicts bistability of patterns. Where there is bistability, the observed pattern will be dependent on the path taken through parameter space and might consist of competition between the different possible stable states. Consequently, in some cases it is only possible to say that the weakly nonlinear theory is consistent with experiments.

The results suggest that the qualitative idea that the three-wave resonances determine the angles that appear in the patterns does indeed explain many of the patterns observed close to onset.

The results also highlight the particular aspects of the Faraday problem that lead to an amplification of $b_{\text {res }}$. Equation (4.4),

$$
b\left(\theta_{\text {res }}\right)=b_{0}+b_{\text {res }}, \quad b_{\text {res }}=-\frac{\alpha_{1} \alpha_{2}}{\lambda_{2}},
$$

suggests that there are two main contributions, one from the quadratic coupling coefficients $\left(\alpha_{1}\right.$ and $\left.\alpha_{2}\right)$ and one from the linear damping of the weakly damped mode $\left(\lambda_{2}\right)$. Promotion of patterns with angle $\theta_{\text {res }}$ requires the quadratic coefficients to be sufficiently large and/or the damping to be sufficiently small. As illustrated in figure 13(a) and (b) for $j=\{4,5\}$ and in (d) and (e) for $j=\{4,5,2\}$ although increasing $a_{5}$ and heading towards the bicritical point would appear to change the value of the linear damping for the $2 \omega / 2$ tongue, this is in fact not the dominant effect. This can be seen in figure 13 (c) and also in (f) where the Floquet multipliers are plotted for both $a_{5}=0$ and a value of $a_{5}$ near the bicritical point. It can be seen that increasing the amplitude of the $j=5$ mode in the forcing promotes the $5 \omega / 2$ tongue, as expected, but has little impact on the damping of the other modes. In contrast, now comparing figure 13(c) with (f), we see that the addition of the $j=2$ mode does have the effect of reducing the damping of the mode associated with the $2 \omega / 2$ tongue as seen by an increase in the Floquet Multiplier from approximately 0.5 for $a_{2}=0$ to 0.9 for $a_{2}=0.8 \mathrm{~g}$.

Rather than changing the damping of the weakly damped mode, increasing the amplitude of the $j=5$ mode, so heading towards the bicritical point, has the effect of increasing the quadratic coefficients $\alpha_{1}$ and $\alpha_{2}$ : in the limit when $a_{5}=0$ the quadratic coefficients are zero. This is because the temporal resonance condition discussed in section 4.1 is not met when $a_{5}=0$, so there can be no coupling between the $2 \omega / 2$ mode and the $4 \omega / 2$ mode.

Topaz et al. (2004) consider a weak viscosity limit and show that in the case of $j=\{m, n\}$ forcing the temporal resonance condition results in possible three-wave interactions with an $(n-m)$ mode, where one expects

$$
b_{\mathrm{res}}=\frac{\alpha\left|a_{n}\right|^{2}}{\left|\lambda_{2}\right|},
$$

where $\lambda_{2}$ is the linear damping of the weakly damped mode and $\alpha$ is a coefficient. Although this cannot necessarily be expected to apply to the moderate viscosity case of the experiments in Ding \& Umbanhowar (2006), we see in figure 14 that the calculated values for $b_{\text {res }}$ for the $j=\{4,5\}$ case have a close to quadratic dependence on $a_{5}$ as suggested by equation (6.1). For $j=\{m, n, p\}$ frequency forcing that has a three-wave resonance with an $(n-m)$ mode, as for the case $j=\{4,5,2\}$, Topaz et al. (2004) find 
(a)

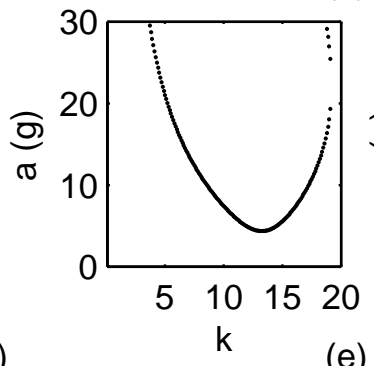

(d)

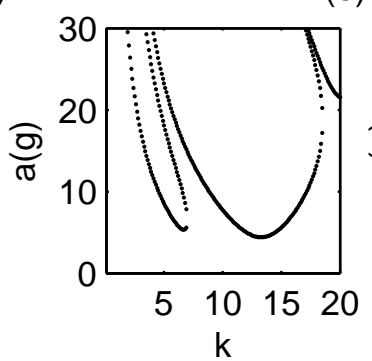

(b)
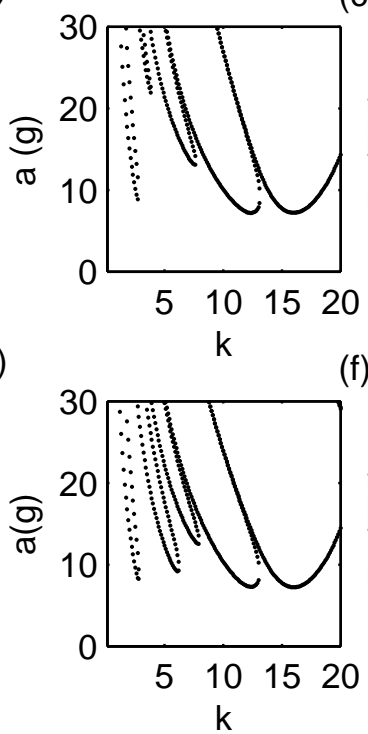

(c)

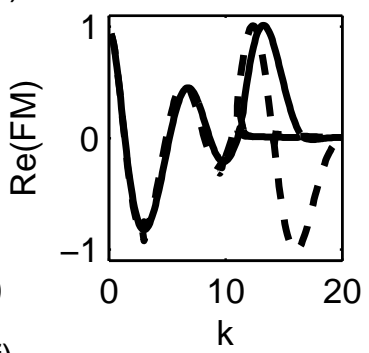

(1)

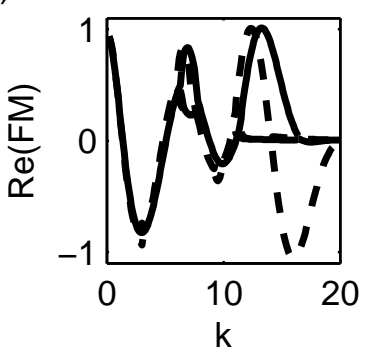

FiguRE 13. Linear stability curves and Floquet multipliers for $j=\{4,5\}$ and $j=\{4,5,2\}$ excitation using parameter values as in Ding \& Umbanhowar (2006). (a) and (b) Linear stability curves for $f_{d}(t)=a(\cos \chi \cos 4 \omega t+\sin \chi \cos 5 \omega t)$, where (a) has $\chi=0$ and (b) has $\chi=63^{\circ}$. (c) the corresponding Floquet multipliers where the solid line is for $\chi=0$ and the dashed line is for $\chi=63^{\circ}$. (d) and (e) Linear stability curves for $f_{d}(t)=a\left(\cos \chi \omega t+\sin \chi \cos 5 \omega t+\tilde{a}_{2} \cos 2 \omega t\right.$ where (a) has $\chi=0, a_{2}=a \tilde{a}_{2}=0.8 \mathrm{~g}$ and (b) has $\chi=62^{\circ}, a_{2}=a \tilde{a}_{2}=0.8 \mathrm{~g}$. (c) The corresponding Floquet multipliers where the solid line is for case (d) where $a_{5}=0$ and the dashed line is for (e) where $a_{5} \neq 0$.

that the dominant contribution to $b_{\text {res }}$ is given by

$$
b_{\text {res }}=\alpha\left|a_{n}\right|^{2} P_{2}(\Phi),
$$

where $\Phi=\phi_{p}+2 \phi_{m}-2 \phi_{n}$ and $P_{2}$ is given by

$$
P_{2}=\frac{\left|\lambda_{2}\right|+\mu a_{2} \sin \Phi}{\left|\lambda_{2}\right|^{2}-\mu^{2} a_{2}^{2}},
$$

where $\mu$ is a coefficient. As $a_{2} \rightarrow 0$, equation 6.2 reduces to equation 6.1). However, for $a_{2}$ non-zero, the effect of $P_{2}$ is to make the leading order dependence of $b_{\text {res }}$ on $a_{2}$ not purely quadratic, so it is not surprising that for this case, an assumption of quadratic dependence fits less well.

Note that the $\Phi$ dependence, which comes from a parameter symmetry, does explain the strong diagonal structure to the figures showing the pattern dependence as a function of two of the phases, see figures 9 and 12

There is a further issue to consider. Increasing the amplitude of the $j=2$ mode brings the location of the bicritical point where both the $4 \omega / 2$ and the $2 \omega / 2$ mode onset simultaneously closer to the pattern onset point. This is seen in figure 7 which shows both the linear stability boundaries for both $4 \omega / 2$ and $2 \omega / 2$ modes. The proximity of the linear stability boundary means that, in order to quantitatively predict the regions of quasipatterns, it is likely that one would need to consider the tri-critical problem where $4 \omega / 2,5 \omega / 2$ and $2 \omega / 2$ modes all occur simultaneously. The onset boundaries for the three modes for $j=\{4,5,2\}$ are shown in figure 15 the position of the tri-critical 


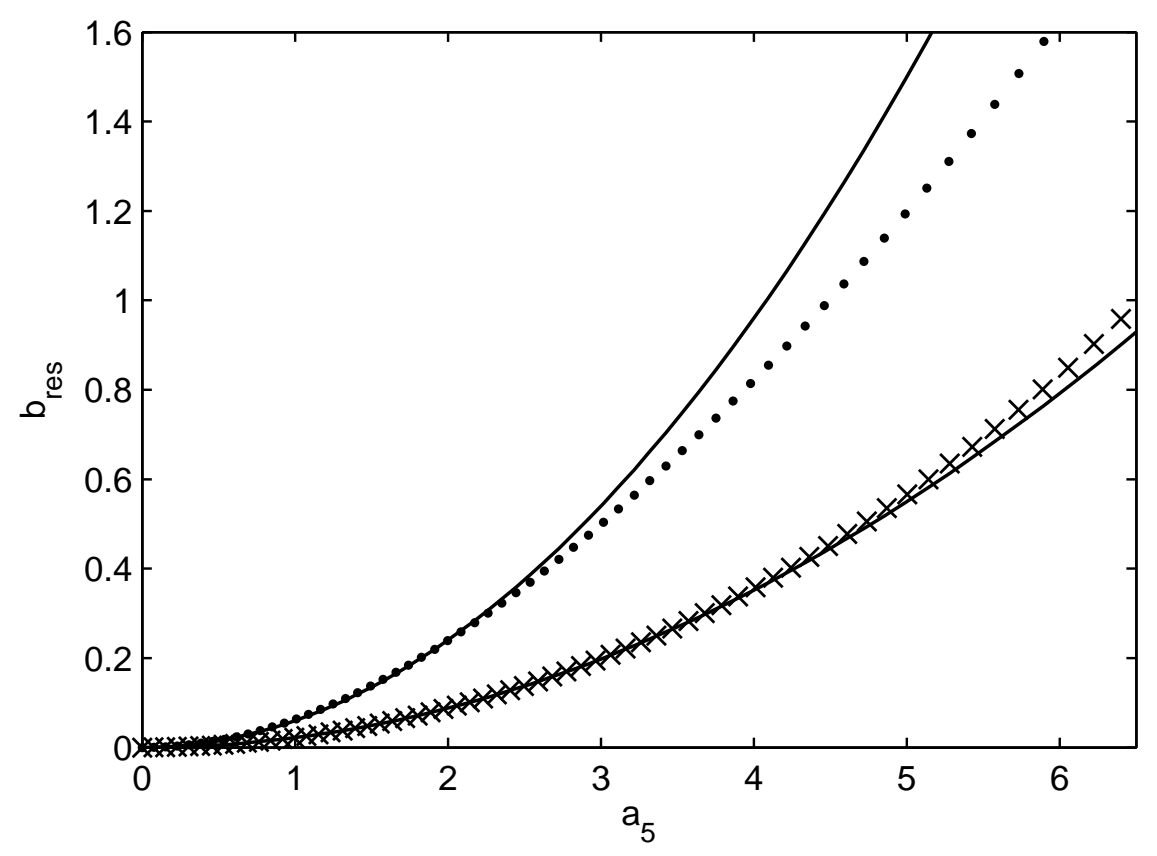

Figure 14. The value of $b_{r e s}$ for $j=\{4,5\}$ (crosses) and for $j=\{4,5,2\}$ (dots) for increasing $a_{5}$. The solid lines are proportional to $a_{5}^{2}$.

point is at approximately $\left(a_{4}, a_{4}, a_{2}\right)=(3.5,6.4,1.0)$. The situation for $j=\{6,7,2\}$ forcing is similar, and one explanation for discrepancies between the weakly nonlinear theory and experimental results may be that they are a consequence of the proximity of the codimension three point.

Another important feature of the analysis considered here is that, the codimension one description of the problem results in amplitude equations that are variational. This variational structure is useful and it enables us to construct a Lyapunov functional to predict the 'most stable' pattern. However, this variational framework also means that all predicted patterns repeat with either the period or half the period of the drive but cannot exhibit more complicated time dependence. As discussed in Rucklidge et al. (2012), in the vicinity of the bicritical point and depending on the signs of the quadratic terms, an infinite sequence of resonances can take place leading to spatio-temporal chaos. This may explain some of the disordered states seen close to onset in the experiments in Epstein \& Fineberg (2004) and Ding \& Umbanhowar (2006). The difficulties of analysing and numerically solving the time-dependent Navier-Stokes equations leaves a clear role for reduced models here. For example, the model of Zhang \& Viñals (1997) is derived from the Navier-Stokes equations under the assumptions of infinite depth and weak viscosity but, as was shown in Skeldon \& Porter (2011), weakly nonlinear analysis of the Zhang-Vinãls equations agrees surprisingly well with weakly nonlinear analysis of the full Navier-Stokes equations even for the moderate viscosity values used in many Faraday experiments.

Finally, our results suggest that weakly nonlinear theory is a useful tool to understand the pattern transitions near onset, but there are some caveats. Ideally experiments and theory would be done hand-in-hand otherwise it may be hard to get agreement even in the linear theory, as we saw in section 3; or establish which modes are present in patterns, as we saw in section 4 ; or establish if there is bistability, as we saw in section 5 . There are 


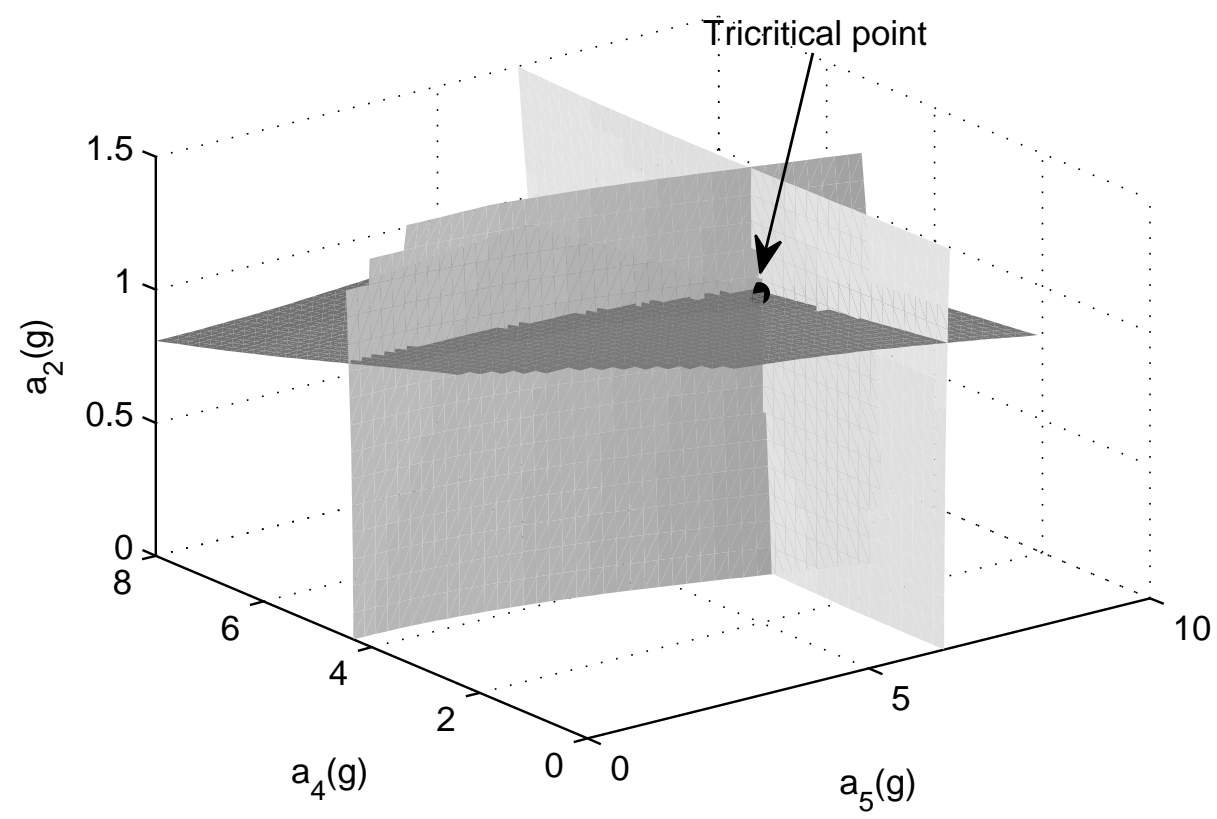

FiguRE 15. Surfaces for the onset of modes with frequencies $4 \omega / 2,5 \omega / 2$ and $2 \omega / 2$.

even problems at the basic level of the definition of the forcing in equation (2.1). In single frequency experiments changing the sign of $f(t)$ is equivalent to a phase shift in time, so the sign of the forcing is not important. However, for multiple frequencies the analogous result is perhaps less transparent: changing the sign requires not just a translation in time but requires an altered definition for the phases. The particular sign convention used by any one experimental group will depend on how the accelerometer is wired up to the experiment. Specifically, it will depend on whether or not the accelerometer records its maximum value when the oscillator is at its highest point or at its lowest. This level of detail is not normally recorded - perhaps because an assumption is made that it does not matter. However, this can lead to some confusion: for example, the phase invariant $\Phi$ discussed in Porter et al. (2004) is derived with one particular choice of sign for $f(t)$ and thus one specific definition of the phases for the forcing components. Ding \& Umbanhowar (2006) note that they find a difference between the position of the maximum value of the phase invariant and the theory of Porter et al. (2004) and between their results and those of Epstein \& Fineberg (2006), but in both cases these differences can be explained by a different convention for the sign of the forcing $f(t)$. 


\section{Appendix A. Calculating Floquet multipliers}

The Floquet multipliers can be calculated by considering the linear stability of the trivial solution (2.9) of equation (2.6) and associated boundary conditions to perturbations of the divergence free form

$$
\mathbf{u}=\left(\frac{i}{k} \partial_{z} W(z, t), 0, W(z, t)\right) \mathrm{e}^{i k x} .
$$

This leads to a fourth order equation for $W(z, t)$ for $-h / l<z<0$

$$
\left(\partial_{t}-C\left(-k^{2}+\partial_{z z}\right)\right)\left(-k^{2}+\partial_{z z}\right) W=0,
$$

with boundary conditions at $z=-h / l$,

$$
W(-h / l, t)=\left.\partial_{z} W\right|_{-h / l}=0
$$

and at $z=0$,

$$
\begin{aligned}
\partial_{t} Z & =W \\
\left(k^{2}+\partial_{z z}\right) W & =0 \\
\left(\partial_{t}+C\left(3 k^{2}-\partial_{z z}\right)\right) \partial_{z} W & =-\left((1+a f(t)) k^{2}+B k^{4}\right) Z
\end{aligned}
$$

A finite difference discretization of equation (A 1) and its boundary conditions is then carried out by letting $W_{j}^{n}=W\left(z_{j}, t_{n}\right), j=0 . . J, z_{j}=-h / l+h j / l J$ and $t_{n}=n \delta t$ and $Z^{n}=Z\left(t_{n}\right)$. The resulting map is of the general form

$$
\mathbf{A}\left(\begin{array}{c}
W_{1}^{n+1} \\
W_{2}^{n+1} \\
\vdots \\
W_{J-1}^{n+1} \\
W_{J}^{n+1} \\
Z^{n+1}
\end{array}\right)=\mathbf{B}\left(t_{n}\right)\left(\begin{array}{c}
W_{1}^{n} \\
W_{2}^{n} \\
\vdots \\
W_{J-1}^{n} \\
W_{J}^{n} \\
Z^{n}
\end{array}\right),
$$

where $\mathbf{A}$ and $\mathbf{B}$ are $(J+1) \times(J+1)$ matrices given by

$$
\mathbf{A}=\left(\begin{array}{ccccccc}
\tilde{a} & 1 & 0 & & & & \\
1 & \tilde{a} & 1 & 0 & & & \\
0 & 1 & \tilde{a} & 1 & 0 & & \\
\vdots & & & \ddots & & & \vdots \\
& & 0 & 1 & \tilde{a} & 1 & 0 \\
& & & 0 & 2 & \tilde{a} & 0 \\
& & & & & 0 & 1
\end{array}\right)
$$

and

$$
\mathbf{B}=\left(\begin{array}{ccccccccc}
c+C \delta t / \delta z^{2} & b & C \delta t / \delta z^{2} & 0 & & & & & \\
b & c & b & C \delta t / \delta z^{2} & 0 & & & & \\
C \delta t / \delta z^{2} & b & c & b & C \delta t / \delta z^{2} & 0 & & & \\
0 & C \delta t / \delta z^{2} & b & c & b & C \delta t / \delta z^{2} & 0 & & \\
\vdots & & & & \ddots & & & & \vdots \\
& & 0 & C \delta t / \delta z^{2} & b & c & b & C \delta t / \delta z^{2} & 0 \\
& & & & C \delta t / \delta z^{2} & b & c-C \delta t / \delta z^{2} & b+d & 0 \\
& & & & & 2 C \delta t / \delta z^{2} & b+e & c+g & \tilde{f}^{n} \\
& & & & & & 0 & \delta t & 1
\end{array}\right) .
$$


where

$$
\begin{aligned}
\tilde{a} & =-\left(2+k^{2} \delta z^{2}\right) \\
b & =1-2 C k^{2} \delta t-4 C \frac{\delta t}{\delta z^{2}} \\
c & =-\left(2+k^{2} \delta x^{2}\right)+C \frac{\delta t}{\delta z^{2}}\left(6+4 k^{2} \delta z^{2}+k^{4} \delta z^{4}\right) \\
d & =C \frac{\delta t}{\delta z^{2}}\left(2-k^{2} \delta z^{2}\right) \\
e & =1-4 C k^{2} \\
\tilde{f}^{n} & =2 \delta t \delta z\left(\left(1+f\left(t_{n}\right)\right) k^{2}+B k^{4}\right) \\
g & =-C \frac{\delta t}{\delta z^{2}}\left(2-k^{2} \delta z^{2}\right)^{2} .
\end{aligned}
$$

The matrix $\mathbf{B}$ contains time dependence through the term $\tilde{f}^{n}\left(t_{n}\right)$.

The map A 2 is iterated through one period, $T$, of the drive resulting in a map $\mathbf{W}^{N}=\mathbf{D W}^{0}$ that takes $\mathbf{W}^{0}$ to $\mathbf{W}^{N}$ where $N=T / \delta t$. The eigenvalues of the matrix $\mathbf{D}$ then give the required Floquet multipliers.

This paper builds on discussions about pattern formation in the Faraday problem with many individuals over the years. In particular the authors would like to acknowledge the input of Mary Silber, Jeff Porter and Jay Fineberg.

\section{REFERENCES}

Alnahdi, A.S., Niesen, J. \& Rucklidge, A.M. 2014 Localized patterns in periodically forced systems. SIAM Journal on Applied Dynamical Systems 13, 1311-1327.

Arbell, H. \& FineberG, J. 1998 Spatial and temporal dynamics of two interacting modes in parametrically driven surface waves. Physical Review Letters 81, 4384-4387.

Arbell, H. \& Fineberg, J. 2000 Temporally harmonic oscillons in Newtonian fluids. Physical Review Letters 85, 756-759.

Arbell, H. \& Fineberg, J. 2002 Pattern formation in two-frequency forced parametric waves. Physical Review E 65, 036224.

Benjamin, T. B. \& Ursell, F. 1954 The stability of a plane free surface of a liquid in vertical periodic motion. Proceedings of the Royal Society, London A 225, 505-515.

Besson, T., Edwards, W. S. \& Tuckerman, L. S. 1996 Two-frequency parametric excitation of surface waves. Physcial Review E 54, 507-513.

Bodenschatz, E., Pesch, W. \& Ahlers, G. 2000 Recent developments in Rayleigh-Bénard convection. Annual Reviews of Fluid Mechanics 32, 709-778.

Christiansen, Bo, Alström, Preben \& Levinsen, Mogens T. 1992 Ordered capillary-wave states: Quasicrystals, hexagons, and radial waves. Physical Review Letters 68, 2157-2160.

Ding, Y. 2006 Pattern formation in multi-frequency driven fluid surface waves.

Ding, Y. \& Umbanhowar, P. 2006 Enhanced Faraday pattern stability with three-frequency drive. Physical Review E 73, 046305.

Dionne, B., Silber, M. \& Skeldon, A. C. 1997 Stability results for steady, spatially periodic planforms. Nonlinearity 10, 321-353.

EDWARDS, W. S. \& FAuve, S. 1994 Patterns and quasi-patterns in the Faraday experiment. Journal of Fluid Mechanics 278, 123-148.

Epstein, T. \& FineberG, J. 2004 Control of spatiotemporal disorder in parameterically excited surface waves. Physical Review Letters 92, 244502.

Epstein, T. \& Fineberg, J. 2006 Grid states and nonlinear selection in parametrically excited surface waves. Physical Review E 73, 055302. 
FARADAY, M. 1831 On the forms and states of fluids on vibrating elastic surfaces. Philosophical Transactions of the Royal Society London 121, 319-340.

Iooss, G. \& Rucklidge, A.M. 2010 On the existence of quasipattern solutions for the SwiftHohenberg equation. Journal of Nonlinear Science 20, 361-394.

Kahouadji, L., Périnet, N., Tuckerman, L. S., Shin, S., Chergui, J. \& Juric, D. 2015 Numerical simulation of super-square patterns in faraday waves.

Kudrolli, A., Pier, B. \& Gollub, J. P. 1998 Superlattice patterns in surface waves. Physica D 123, 99-111.

Kumar, K. \& Tuckerman, L. S. 1994 Parametric instability of the interface between two fluids. Journal of Fluid Mechanics 279, 49-68.

Lifshitz, R. \& Petrich, D. M. 1997 Theoretical model for Faraday waves with multiplefrequency forcing. Physical Review Letters 79, 1261-1264.

Malkus, M. V. R. \& Veronis, G. 1958 Finite amplitude cellular convection. Journal of Fluid Mechanics 4, 225-260.

Melbourne, I. 1999 Steady-state bifurcation with Euclidean symmetry. Transactions of the American Mathematical Society 351, 1575-1603.

Müller, H. W. 1994 Model equations for two-dimensional quasipatterns. Physical Review E 49, $1273-1277$.

Périnet, Nicolas, Juric, Damir \& Tuckerman, Laurette 2012 Alternating hexagonal and striped patterns in Faraday surface waves. Physical Review Letters 109, 164501.

Porter, J. \& Silber, M. 2002 Broken symmetries and pattern formation in two-frequency forced Faraday waves. Physical Review Letters 89, 054501.

Porter, J., Topaz, C. M. \& Silber, M. 2004 Pattern control via multifrequency parametric forcing. Physical Review Letters 93, 034502.

Rogers, J. L., Schatz, M. F., Brausch, O. \& Pesch, W. 2000 Superlattice patterns in vertically oscillated Rayleigh-Bénard convection. Physical Review Letters 85, 4281-4284.

Rucklidge, A. M. \& Rucklidge, W. J. 2003 Convergence properties of the 8, 10 and 12 mode representations of quasipatterns. Physica D 178, 62-82.

Rucklidge, A. M. \& Silber, M. 2009 Design of parametrically forced patterns and quasipatterns. SIAM Journal on Applied Dynamical Systems 8, 298-347.

Rucklidge, A. M., Silber, M. \& Skeldon, A. C. 2012 Three-wave interactions and spatiotemporal chaos. Physical Review Letters 108, 074504.

Segel, L. A. \& Stuart, J. T. 1962 On the question of the preferred mode in cellular thermal convection. Journal of Fluid Mechanics 13, 289-306.

Silber, M. \& Proctor, M.R.E. 1998 Nonlinear competition between small and large hexagonal patterns. Physical Review Letters 81, 2450-2453.

Silber, M. \& Skeldon, A. C. 1999 Parametrically excited surface waves: Two-frequency forcing, normal form symmetries, and pattern selection. Physical Review E 59, 5446-5456.

Silber, M., Topaz, C. \& Skeldon, A. C. 2000 Two-frequency forced Faraday waves: weakly damped modes and pattern selection. Physica D 143, 205-225.

Simonelli, F. \& Gollub, J.P. 1989 Surface wave mode interactions: effects of symmetry and degeneracy. Journal of Fluid Mechanics 199, 471-494.

Skeldon, A. C. \& Guidoboni, G. 2007 Pattern selection for Faraday waves in an incompressible viscous fluid. SIAM Journal on Applied Mathematics 67, 1064-1100.

Skeldon, A. C. \& Porter, J. 2011 Scaling properties of weakly nonlinear coefficients in the faraday problem. Physical Review E 84, 016209.

Skeldon, A. C. \& Silber, M. 1998 New stability results for patterns in a model of longwavelength convection. Physica D 122, 117-133.

Topaz, C., Porter, J. \& Silber, M. 2004 Multifrequency control of Faraday wave patterns. Physical Review E 70, 066206.

ZhANG, W. \& ViÑALS, J. 1997 Pattern formation in weakly damped parametric surface waves. Journal of Fluid Mechanics 336, 301-330. 